\title{
Chapitre 15 Variables en jeu dans le travail de bords
}

Le parcours de variables esquissé ci-dessous répond, on l'a dit, au plan discursif, à celui sur lequel s'achevait le chapitre 9 , au plan des formes de langue : mais, s'il en partage le caractère programmatique, le changement de plan, de la langue vers le discours, implique des différences profondes.

La « grille » de 7 variables, énumérées ci-dessus, proposée comme « outil » pour avancer dans l'appréhension de la diversité des Discours quant à leur rapport aux autres discours relève d'un tout autre statut que le système de distinctivité - de trois traits pertinents combinés en cinq formules de modes - mis au jour comme régissant, en langue, le champ de la RDA. Ces variables correspondent à des paramètres, envisagés en fonction de leur pertinence - pris ici au sens d'efficacité descriptive. Leur jeu combiné n'a nullement le caractère d'un système distinctif clos : d'autres variables pourraient certainement être prises en compte ; chacune d'entre elles, loin de présenter une opposition discrète, ouvre sur le continuum d'un espace de variation. Elles sont de natures hétérogènes, quantifiables pour certaines, en termes de degré entre deux pôles (Étendue $\left(\mathrm{V}_{1}\right)$, taux de Dispersion $\left(\mathrm{V}_{2}\right)$ )...), non pour les autres. Telles, par exemple, celle $\left(\mathrm{V}_{5}\right)$ de la nature et de la tonalité du Rapport établi entre le Discours et ses autres, ou celle $\left(\mathrm{V}_{6}\right)$ des Formes de RDA par lesquelles s'opèrent le contact et le partage entre ceux-ci dans la bordure... Est en cause ici la distinction que Milner formule ${ }^{1}$ entre, d'un côté, « un réel [...] marqué de discernable », un objet [...] à coup sûr structuré, « intrinsèquement pourvu de discernements », celui de la langue, et de l'autre - celui précisément de nos variables - propre notamment aux disciplines herméneutiques, « un flux où sont introduites des coupures », sur le mode d'une " territorialisation opérée à des fins de connaissance ».

Chacune de ces variables demande un balisage attentif de l'espace dans lequel elle déploie son jeu, ce que - touchant à son terme - le parcours entrepris par cet ouvrage ne permet pas d'envisager. Cependant, en s'appuyant notamment à la richesse des acquis descriptifs du « discours rapporté », tant dans le cadre des analyses de discours que dans celui des études littéraires, il est possible d'accompagner de quelques exemples la démarche proposée, ajoutant à la perspective de « prendre les Discours par leurs bords » la conviction qu'il est pertinent d'explorer la diversité de ces bords par les variables qui les dessinent.

1 Milner (1978 : 65-67), (1983 : 39).

ว Open Access. () 2020 Jacqueline Authier-Revuz, published by De Gruyter. (cc)BY-NC-ND This work is licensed under the Creative Commons Attribution-NonCommercial-NoDerivatives 4.0 License. https://doi.org/10.1515/9783110641226-024 
La démarche suivie est, relativement aux Discours perçus comme unité de fonctionnement et de sens, reçue comme un tout, foncièrement dissociatrice : c'est chaque variable dont le jeu est déployé, séparément, par rapport auquel les Discours peuvent être situés. Explorer la diversité des Discours, variable après variable, c'est se donner un principe ou un outil d'analyse, de prise de distance, permettant de reconnaître et de dégager, successivement, dans l'optique de chacune de ces variables, des éléments «normalement » fondus dans la globalité d'un Discours.

Il s'agit donc, dans un premier mouvement, de s'orienter dans le milieu touffu, compact, de la discursivité, par une seule variable à la fois, c'est-à-dire selon sa perspective unique - Étendue, Dispersion, etc. - d'y tracer un chemin au long duquel se « rangent » les Discours.

Le parcours de chacune de ces variables révèle des valeurs extrêmes ${ }^{2}$, des continuums $^{3}$, des pondérations ${ }^{4} . .$. ; fait apparaître des regroupements ${ }^{5}$ inattendus de Discours en fonction de leur placement commun dans l'espace d'une ou plusieurs variables - propres à des types de discours ; fait émerger enfin des affinités $^{6}$ entre valeurs de variables ${ }^{7}$.

Avec ses effets de classement, de tri, d'organisation, le passage de la réalité discursive par la grille différenciatrice de ces sept variables débouche, dans un deuxième temps, sur la possibilité d'envisager, pour chaque Discours, la réponse propre, multicaractérisante, que, dans sa bordure, il apporte à autant de questions sur son « être avec ».

Ces sept variables ne font pas un ensemble homogène : on peut y reconnaître trois groupes. Celui [I] des deux premières qui, touchant l'Étendue $\left(\mathrm{V}_{1}\right)$ occupée dans un Discours par sa bordure et le taux de Dispersion $\left(V_{2}\right)$ des autres qui y sont représentés, se situe en deçà du groupe [II] des variables $\mathrm{V}_{3}$ à $\mathrm{V}_{6}$ concernant individuellement chacune des occurrences de RDA du Discours (degré d'identification de l'autre, forme mise en œuvre, tonalité du rapport D/d...), tandis que

2 Discours à forme unique $\left(\mathrm{V}_{6}\right)$ de $\mathrm{RDA}$, par exemple, ou à centration $\left(\mathrm{V}_{2}\right)$ maximale sur un autre unique...

3 Dégradé de l'Étendue $\left(\mathrm{V}_{1}\right)$ de RDA « de l'objet » $\left(\mathrm{V}_{4}\right)$ dans des Discours consacrés à une personne (portrait, biographie...), un pays étranger (guide touristique, récit de voyage...).

4 Entre spécification et non-spécification $\left(\mathrm{V}_{3}\right)$ des autres convoqués dans un même Discours.

5 Le procès verbal et le pastiche (entre autres) comme genres extrêmes « tout en RDA » $\left(\mathrm{V}_{1}\right)$.

6 Entre Dispersion $\left(V_{2}\right)$ forte et Émergence $\left(V_{4}\right)$ comme associé, par exemple, ou entre Étendue $\left(\mathrm{V}_{1}\right)$ maximale et homogénéité des Formes $\left(\mathrm{V}_{6}\right)$ mises en œuvre.

7 Les liens observés - configuration, affinité - sont posés entre variables autonomes, en ce sens qu'aucune ne dépend strictement d'une autre (ce qui conduirait à les fusionner). 
$\mathrm{V}_{7}$ se détache des groupes I et II par la dimension temporelle qu'elle introduit, concernant toutes les variables.

Ce qui suit présente un parcours des variables $\left(\mathrm{V}_{1}\right)$ Étendue, $\left(\mathrm{V}_{2}\right)$ Dispersion, $\left(\mathrm{V}_{3}\right)$ Spécification et $\left(\mathrm{V}_{7}\right)$ Mouvement de la RDA dans les Discours ; les variables

$\left(V_{4}\right)$ Émergence du DA comme attaché/associé, $\left(V_{5}\right)$ Rapport entre le Discours et son ailleurs, $\left(\mathrm{V}_{6}\right)$ Forme de la $\mathrm{RDA}$, constamment « croisées » dans le parcours de $\mathrm{V}_{1}, \mathrm{~V}_{2}, \mathrm{~V}_{3}$, ne sont pas envisagées ici pour elles-mêmes. Le point 4, p. 622, revient brièvement sur ce choix.

\section{Variable $\left(V_{1}\right)$ : Étendue de la bordure}

Entre les deux pôles où la notion même de bordure perd son sens, dans des discours ignorant la zone interface dedans/dehors, la variation quant à la place qu'un Discours consacre à l'accueil de « ses » autres est aussi importante que significative dans l'économie des discours.

La taille du territoire qu'un Discours assigne à sa bordure de RDA est certes en rapport avec le nombre d'occurrences de RDA mais ne se confond pas avec elles : d'amples coulées, peu nombreuses, de DD, de Bivocal ou de MAS peuvent occuper un large espace tandis qu'un Discours peut, sur une surface étroite, multiplier des MAE à incidence ponctuelle...

Le survol de cet espace $\left(V_{1}\right)$ de variation - à détermination fortement mais non exclusivement générique - passera par le repérage - non exhaustif - de discours à présence maximale (1.1) vs minimale (1.2) de RDA, avant de noter la pertinence différenciatrice du « taux » de RDA dans les discours de plusieurs champs ou domaines (1.3), pour rappeler, enfin, la radicale diversité, au plan singulier, de la taille des bordures dont s'entoure une parole, un style...

\subsection{Discours tout « en RDA »}

C'est un « continent » que celui des discours relevant intégralement de la RDA - à ne pas confondre avec les Discours tout entier livrés à, ou traversés par, l'Autre ou l'ailleurs du langage ${ }^{8}$. On peut y reconnaître, caractérisables au plan générique, plusieurs secteurs.

8 La quête de Flaubert - celle d'un $\mathbf{L}$ « invisible mais présent partout » dans un discours qui semble se faire « tout seul » - se situant au point de bascule entre les deux. 
L'extrême diversité de cet ensemble de Discours à bordure maximale appelle quelques remarques :

- elle témoigne de l'indépendance des variables les unes par rapport aux autres : une bordure occupant une place si grande dans le Discours que celui-ci semble se faire tout entier « support de discours autres » ne prédit pas pour autant la valeur des autres variables ;

- elle appelle l'étude des formes variées par lesquelles se manifeste la présence surplombante du L énonçant, de fait (procès-verbal par exemple) ou fictivement (roman), la totalité du Discours ;

- elle débouche sur les limites assignées à cet ensemble de Discours « de RDA », posant, notamment, la question du statut de «tenant lieu » du texte traduit et du texte théâtral ( $c f$. Remarque 1 p. 597).

\subsubsection{Discours « tenant lieu » d'un autre discours.}

Dans cet espace générique, qu'un parcours vise à définir dans la masse des Discours à RDA maximale ${ }^{9}$, le mode sur lequel s'établit le rapport de « lieu-tenance » entre deux discours dont l'un, en représentant l'autre, "vaut pour lui » est divers.

Ainsi, un important sous-ensemble confère au rapport de « tenant lieu » un statut légal, juridique, d'attestation (que marquent signatures, votes d'approbation...) et comporte une prescription quant à la forme (DD, DI) mise en œuvre dans le texte ${ }^{10}$ : procès-verbaux, compte rendus, rapports de « faits de parole » - réunion de co-propriétaires, soutenance de thèse, conseil universitaire, municipal, d'entreprise, séance de l'Assemblée Nationale (figurant au Journal officiel), d'organisme international, déposition à la police, minute de procès ${ }^{11}$, d'interrogatoire, etc.

Ces discours partagent, associé au statut légal de leur « lieu-tenance », le fait de reposer sur une transcription, et donc sur une reformulation : celle-ci allant de

9 Authier-Revuz et Lefebvre (2015), parallèlement à Sitri (2015b), investigation poursuivie dans un colloque : « L'espace générique des discours “tenant lieu“ d'un autre discours et le champ de la représentation du discours autre », 13-15 novembre 2017, co-organisé par Modyco (Paris-Nanterre) et Clesthia (Paris 3), actes à paraître.

10 Dont la variation, tant synchronique - selon les instances de parole et leur importance institutionnelle (cf. Sitri, 2015b) - que diachronique, d'élaboration de normes au cours de l'histoire ( $c f$. Mazzuchetti, à paraître), s'avère passionante à étudier.

11 À comparer aux Souvenirs de la Cour d'Assises de Gide dont la forte densité de RDA se combine à la description des lieux, aux sentiments de l'écrivain... ou, dans un tout autre style, à la chronique judiciaire du Canard enchaîné, titrée « Coups de barre », croquant des scènes de tribunal à travers des DD, pris sur le vif, assortis de quelques indications « didascaliques ». 
la restitution la plus scrupuleuse, moyennant quelques « lissages » formels pour le travail des « rédacteurs » de l'Assemblée Nationale, à la production d'un texte écrit institué comme équivalent à l'oral qu'il remplace. Le cas des procès-verbaux de déposition auprès d'un fonctionnaire de police pousse à l'extrême le paradoxe - paraphrastique - du différent valant pour du même : c'est en effet un texte précédé d'un l qui nous déclare : « ... », relevant du DD le plus « en substance » qui soit, c'est-à-dire traduit dans un langage administratif que le citoyen $\boldsymbol{l}$ ne partage pas - parfois ignore, parfois même réprouve - et qu'il est amené à " valider », signant ainsi de son nom propre ce qui « tient lieu» de sa parole $\mathrm{p}^{12}$.

Le genre, massivement représenté dans la presse ou le livre, de l'entretien, dans lequel un $\mathbf{L}$ restitue, au DD, l'échange oral, dissymétrique, que lui a accordé un $\boldsymbol{l}$, fait aussi partie de l'espace du " tenant lieu ${ }^{13}$. Sur le fond de l'inévitable transcription, les scrupules, les réécritures ${ }^{14}$, les désinvoltures des $\mathbf{L}$, les méfiances, les précautions, les colères, les exigences de relecture des $\boldsymbol{l}$ jalonnent les parcours d'élaboration de ces «tenant lieu ${ }^{15}$.

Notons le goût, non démenti depuis Fontenelle et ses Entretiens sur la pluralité des mondes, dans le champ de la vulgarisation scientifique, pour le « tenant lieu » fictif d'une conversation entre un savant et une ignorante à l'esprit vif...

Avec tout ce qui relève - ou s'apparente ${ }^{16}$ - au genre du résumé, c'est-àdire d'un couplage paraphrastique de deux textes, effectué cette fois " modulo » une opération de réduction (souvent étroitement normée au plan quantitatif), on aborde des discours du tenant lieu qui, mis pour un discours autre qu'ils représentent, ne sont pas pour autant, contrairement aux genres évoqués précédemment, constitués d'une succession de formes de RDA. Institué comme tel par une annonce ou un titre explicite (résumé, abstract...) et/ou une disposition typographique (caractères, place détachée dans un ensemble plus vaste...), ce genre « de la RDA », affichant son caractère second par rapport au discours autre qu'il « remplace », peut, paradoxalement, selon les contextes institutionnels ou discursifs, comporter ou non des formes de RDA. Entre prescriptions strictes et usages

12 Source d'expériences disphoriques parfois, ce paradoxe est aussi un ressort comique exploité par exemple au cinéma dans Quai des Orfèvres (cf. aussi Eigenmann (1996 : 183) analysant sa mise en œuvre par Vinaver).

13 À différencier en cela du genre du « portrait » composé à la suite d'une rencontre qui, si fréquentes qu'y soient les formes de RDA renvoyant à l'objet du portrait, ne relève pas du Discours « mis pour » un autre discours (cf. Mahrer et Tuomarla (2007), Da Cunha et Lefebvre (2018).

14 Cf. Doquet (2018) sur l'élaboration par F. Fau de Culioli (2002).

15 Cf. Authier-Revuz et Lefebvre (2015).

16 Prises de notes à un cours ; exercices de « contraction de textes » (étudiés in Maldidier et Normand, 1982a, 1982b), par exemple. 
affirmés, la variation quant à la mise en œuvre de formes de RDA s'inscrit entre les deux pôles de textes, également aptes à être reconnus (et affichés) comme résumés, où le statut global de RDA passe par la présence répétée de formes marquées de RDA (DI, MAS, MAE notamment) ou, au contraire par leur absence ; ainsi sont également admissibles au titre de « résumé », affiché comme tel, d'un texte :

(1) Dans cet ouvrage (article, discours...), $X$ soutient le point de vue d'une causalité multifactorielle pour le réchauffement climatique. Récusant l'activité humaine comme facteur unique, il revient, en détail, sur l'histoire - longue - des variations climatologiques. Il souligne, en conclusion, l'urgence de repenser sous tous ses aspects une question obscurcie, selon lui, par «les parti-pris et les fantasmes ». (je souligne)

(2) De multiples facteurs sont cause du réchauffement climatique. Celui-ci n'est pas ramenable à la seule activité humaine, mais s'inscrit dans l'histoire longue des variations climatologiques. Il est urgent de repenser sous tous ses aspects une question obscurcie par les parti-pris et les fantasmes.

Là où le premier passe par une description du fait de discours visé, comportant références à ce discours (cet ouvrage, conclusion), à son énonciateur ( $X$, il, selon lui), catégorisation des actes de parole effectués (soutient, récusant, revient en détail...), mention de ses mots (MAE finale), c'est-à-dire se donne, formellement, comme "parlant de » ce discours, le second consiste, lui, en un texte de remplacement de discours qui, " mis pour » et non " parlant de », relève de la paraphrase pure et bannit toute forme de renvoi (RDA) au discours source. Contrairement à (1) dont les formes affichent un rapport de RDA à un autre texte, c'est seulement un cadre générique manifeste qui confère à (2) une statut textuel, global, de RDA : privé de toute référence à celui-ci, (2) apparaîtrait comme propos de $\mathbf{L}$ sur le climat. Sous l'égide de cette appartenance déclarée au genre du résumé de texte, « tout se passe comme s'il n'y avait pas eu de texte premier ${ }^{17}$.

On reconnaît dans le second type (2) le modèle, par exemple, des « Résumés » qui, détachés comme tels, en fin de chapitre - en encadré, en gras... - dans les manuels scolaires étaient donnés à « apprendre par cœur » : tout à fait incongrue - « a-générique » comme on dit agrammatical - serait l'adjonction d'une référence au discours source, à un item du résumé d'un chapitre consacré aux paysans médiévaux ${ }^{18}$ :

(3) $\quad\left({ }^{\star}\right.$ Ce chapitre explique que/ ${ }^{\star}$ Selon ce chapitre) Presque tous les français sont paysans.

17 Maldidier \& Normand (1982b : 88), analysant les effets de vérité objective auxquels se prêtent ces exercices de contraction, par l'effacement des ancrages historiques et subjectifs du discours premier.

18 Le Moyen-Age, coll. Isaac, classe de 4"e, 1958, Hachette, p. 36. 
Dans ce cas c'est comme régime textuel d'énonciation que, par le titre, la RDA est convoquée ${ }^{19}$.

C’est aussi celui des épreuves (résumé, contraction de texte) de concours dont les strictes consignes portent autant sur le taux de réduction requis (nombre de mots, avec 10\% d'écart admis...) que sur la proscription de toute forme de RDA... ${ }^{20}$ pensée alors comme « stigmate » de l'opération, empêchant la « pleine » substitution.

On peut noter que les résumés qui dans l'espace d'un recueil ou d'un numéro de revue redoublent chaque contribution, participent, très majoritairement, du premier modèle. Ainsi, à la fin d'un volume ${ }^{21}$ regroupant 55 contributions, ce sont 51 des résumés (écrits par les auteurs) qui, avec des densités différentes, réfèrent explicitement au discours premier : passant par les désignations déictiques (de co-présence dans le volume) du texte premier, celles de l'énonciateur, de l'acte de parole qu'il effectue (on se propose dans cet article, nous envisageons ici, l'objectif de la présente étude...), ce sont alors, dans ces textes à paragraphe unique, de une de ces formes (souvent alors en tête de résumé), à des occurrences répétées en quatre, cinq... points, voire à chaque phrase du résumé, qui arriment ceux-ci au texte premier.

En revanche, dans les résumés d'ouvrages publiés dans la presse, dans les livres de résumés destinés à tenir lieu des « livres que l'on doit avoir lus »..., dans les manuels d'études littéraires, se dessine tendanciellement une opposition entre résumés de « récits », sans formes de RDA (« Julien Sorel, fils d’un charpentier, devient précepteur... ») et résumés de textes de réflexion (« Montaigne analyse..., décrit..., dit... ») passant par des formes de RDA.

Pour l'ensemble de ces genres du tenant lieu, la valeur maximale de la variable $\left(\mathrm{V}_{1}\right)$ de l'Étendue est associée à l'un des pôles de la variable $\left(\mathrm{V}_{4}\right)$ de l'Émergence du

19 Sur le mode, $c f$. ci-dessus p. 576 où F. Héritier place un « selon eux » en clef modalisante d'une partie de son discours, proche de celui de B. d'Espagnat (2015), indiquant que «pour éviter la lourdeur insupportable devant chaque phrase des tournures "il me semble que", ou "il paraît très vraisemblable que" il doit être entendu que ces expressions « omises presque partout » y figurent implicitement et sont « à restituer par la pensée ».

$20 \mathrm{Cf}$., relevé sur un des nombreux sites offrant leurs services - conseils et modèles - pour la réussite de cet exercice : « en aucun cas vous ne devez utiliser des formules telles que "l'auteur affirme que" ».

21 Lopez-Munoz et al. (2004) : exemples de résumés à une forme de RDA, dans la phrase initiale (articles numérotés 28,51 , dans la table des matières) ; à formes multiplement répétées $(23,36$, $54)$; à chaque phrase $(18,38,39)$; et, relevant du modèle minoritaire sans forme de RDA (5, 30). La même forte prépondérance du modèle 1 s'observe dans les annonces de parutions des éditeurs : $c f$. le catalogue nouveautés $2009 \mathrm{~A}$. Colin (lettres linguistique) : 29 résumés sur 30 comportent « ce/le livre, cet ouvrage, l'auteur », etc. 
discours autre comme " attaché à l'objet du Discours » - ici dans le cas spécifique ou c'est un discours autre qui est lui-même l'objet du Discours.

Remarque 1 : Marges de l'espace du « tenant lieu ». On peut envisager ${ }^{22}$ la traduction comme relevant de la RDA, au titre de genre du tenant lieu, excluant certes, comme certains résumés, toute forme de RDA renvoyant dans le texte second au texte premier - comme le serait, par exemple, une incise en «nous raconte $l$ » dans l'incipit d'un roman -, mais qu'on peut considérer comme explicitée, en tête, par la mention « traduit de... par $\boldsymbol{x}$ » ( $\boldsymbol{x}$ ayant alors le statut de $\mathbf{L})$ et éventuellement accompagnée par une préface et des notes énoncées par ce $\mathbf{L}$-traducteur.

Plus problématique est le statut du texte théâtral ${ }^{23}$ conçu comme tenant lieu écrit, prospectif, de sa « représentation » (au sens théâtral) orale. Un élément à prendre en compte dans cette question est le jeu observable dans des canevas de la Commedia dell'Arte où alternent régulièrement, avec le DD « fixant » les propos des personnages, les représentations au DI, requérant l'improvisation de l'acteur, à partir de formulations synthétiques du type :

(4) Arlequin se retourne vers la maison en faisant des lamentations sur son malheur. Octave survient en disant : [...]. Arlequin lui apprend que la tête lui fait mal, que sa femme est amoureuse d'un gentilhomme qu'elle nomme son cher cœur et que [...]. Octave, persuadé qu'Eulalia lui est infidèle [...] entre dans une violente colère [...]. Arlequin [...] (« Les tapis d'Alexandrie », in Comédie italienne, Club des Libraires de France. ${ }^{24}$.

\subsubsection{Genres de représentation (RDA) de la partie pour le tout}

Il s'agit de l'ensemble, vaste et diversifié, des genres « collecteurs », au DD, d'éléments de discours autres donnés comme valant pour un ailleurs discursif plus large :

- Anthologies, Morceaux choisis, d'un texte, d'un auteur, d'une période, d'un genre, telles les innombrables anthologies de la poésie française « tout entière » ou " populaire » ou « baroque » ou " contemporaine », etc., où la présence de l'auteur (L) se manifeste souvent par, outre les choix qui sont les siens, une préface explicitant ceux-ci et, chemin faisant, des notules sur les auteurs cités, des notes infrapaginales...

- Dictionnaires de proverbes, de paroles célèbres (cf. " pages roses » du Petit Larousse)...

22 Point de vue argumenté par Folkart (1991).

$23 \mathrm{~L}$ auteur, $\boldsymbol{l}_{1}, \boldsymbol{l}_{2} \ldots$ personnages, $\boldsymbol{e}_{1}, \boldsymbol{e}_{2} \ldots$ propos, loc, temps, indications situationnelles.

24 Exemple emprunté aux larges extraits figurant dans le matériel pédagogique élaboré pour l'étude du « discours rapporté » dans le canton de Vaud (cf. Avant-Propos, note 10, p. XVI). 
- Florilèges autour d'un thème, d'un référent, dédiant à Paris, à Londres, à l'amour, aux jardins, à la mer, à l'enfance etc. des bouquets de propos à leur gloire $^{25}$...

- Dictionnaires des citations évoquées par des mots dont le travail - idéologiquement marqué - de constitution-façonnage de la mémoire interdiscursive est passionnant à observer.

Relativement aux autres variables, cet ensemble de genres de la RDA s'inscrit sur le même versant de la variable $\left(\mathrm{V}_{4}\right)$ comme discours autre « attaché » à l'objet du Discours, sur le mode où l'autre représenté l'est comme « partie de » et valant pour « le tout » de cet objet : discours d'un auteur, d'un genre, de l'ensemble - non fini - des discours circulant autour d'un référent ou passant par un mot.

Les deux derniers types font fonction pour les énonciateurs - à la façon d'une mémoire annexe et au risque des contre-sens issus de leur décontextualisation $^{26}$ - de réservoir de discours autres prêts à être " associés » $\left(\mathrm{V}_{4}\right)$ à d'autres Discours. Tout l'ensemble, en revanche, est astreint, formellement à la seule forme $\left(\mathrm{V}_{6}\right) \mathrm{du} \mathrm{DD}$.

\subsubsection{Genres littéraires « de RDA », associé $\left(V_{4}\right)$ à un autre discours}

Comme les genres (1.1.1) de discours « tenant lieu » d'un autre discours, les genres littéraires, très étudiés ${ }^{27}$, du pastiche et de la parodie, ne prennent sens que par rapport au discours-texte autre auquel ils sont « couplés ». Mais loin de remplacer le discours autre comme son tenant lieu, le pastiche prétend prendre place aux côtés de son modèle, à la manière duquel il est écrit ; il relève d'une énonciation modalisée à l'échelle du texte, en MAE non marquée, du type " comme écrirait », « pourrait/aurait pu écrire $\boldsymbol{l}$ » : ainsi les diverses versions de L'Affaire Lemoine, proposées par Proust, sont-elles écrites « en clef de » Flaubert, Balzac, etc.

La parodie, elle, ne cherche pas à faire un " même » à partir d'un modèle, mais, au contraire, à altérer caricaturalement un texte pour en faire un autre : comme pour le pastiche, la RDA qui affecte l'ensemble du texte parodique, de façon non marquée, est une modalisation (MAE) foncièrement prédatrice, glosable en « pour m'emparer du texte de $\boldsymbol{l}$, en le dévoyant à ma fantaisie »... comme

$25 C f$. « Passion de la danse - Citations » évoqué chap. 5.5.1.2, p. 180.

26 Cf. chap. 5.1.2, ex. (71), p. 181.

27 Outre, bien entendu, Genette (1982) pour ces formes de littérature au second degré $c f$. Samoyault (2010 : 33 sq.) et, pour un parcours original et éclairant d'auteurs contemporains, Bikialo (2014). 
le canonique Chapelain décoiffé de Boileau en donne l'exemple dans le traitement qu'il fait subir au Cid:

(5) Ô rage ! ô désespoir : ô perruque ma mie !/N'as-tu donc tant vécu que pour cette infamie ?/N'as-tu trompé l'espoir de tant de perruquiers,/Que pour voir en un jour flétrir tant de lauriers?

ou, dans le roman contemporain, la parole des Évangiles courant tout au long de celle de Tobold, roi du Hamburger, célébrant sa mission d'apporter la frite et le hamburger aux petits enfants ${ }^{28}$.

Les genres (ou styles) « burlesque » et héroïcomique relèvent du même mécanisme de dérivation modalisante d'un texte à partir d'un ailleurs qui est cette fois celui d'un style (manière de dire) autre que celui normalement en usage pour un type de référent - selon une MAE, non marquée, explicitable en « pour parler vulgairement/noblement de choses nobles/vulgaires $»^{29}$.

Un autre genre littéraire « de RDA », le centon, se différencie des précédents par sa forme de RDA $\left(V_{6}\right)$, le DD non marqué et, contrastant avec leur autre - texte, auteur, ton - unique, la Dispersion $\left(\mathrm{V}_{2}\right)$ des autres discours qu'ils mettent en jeu dans la série hétérogène de discours assemblés en " collage », sur le mode du patchwork. Hérité de l'Antiquité, le genre est toujours vivace, réveillant ludiquement, irrespectueusement, lumineusement aussi parfois au gré de rencontres inédites, des mémoires sagement ordonnées. Sans surprise Montaigne l'évoque ${ }^{30}$, Éluard le pratique dans Premières, mes anciennes, J.-B. Pontalis s'y est récemment ${ }^{31}$ adonné, faisant défiler cinquante huit textes, surgis dans « un compartiment de chemin de fer ", petit monde roulant, clos et ouvert à l'imprévu, où, emportés par le mouvement du train-centon, se suivent Michel Strogoff et Lafcadio, Proust et Anna Karénine, Blaise Cendrars et la Famille Fenouillard... ; et c'est comme « naturellement » que les fervents d'une littérature « potentielle » le cultivent... et le raffinent.

28 L. Salvayre, Portrait de l'écrivain en animal domestique, Seuil, Paris, 2007. Cf. notamment les « il dit : Au commencement était l'argent » (p. 123), ou la longue homélie commençant par : « Alors Tobold dit : Les petits enfants, mes amis, sont les anges à notre solde. » (p. 168), et finissant sur : «Les enfants seront les instruments du Nouveau Règne, celui de la frite et du hamburger son ami [...] alleluia » (p. 170).

29 Cf. par exemple, chap. 8 (57), p. 268, « Les Évangiles » des Soliloques du Pauvre de Jehan Rictus, ou ces fables, nombreuses, où La Fontaine déploie à plaisir la Geste... de deux coqs ou celle d'un moucheron, in (Livre II, fable 9 ; Livre VII, fable 12).

30 Livre II 26. Voir l'article Centons du Dictionnaire Montaigne (Ed. Garnier, 2018).

31 Ce temps qui ne passe pas (1997, Folio : 137-202). 
Remarque 2 : Le centon sophistiqué par ordinateur. Rejeton de l'Oulipo, ALAMO (Atelier de Littérature Assistée par la Mathématique et les Ordinateurs ${ }^{32}$ ) est créateur de " programmes » comportant au moins un " corpus » de textes de départ (célèbres alexandrins, La Légende des siècles, Le Dormeur du Val...) et des contraintes d'assemblage : ainsi M. Benabou et J. Roubaud, dans " Alexandrins au greffoir » produisent-ils des vers inédits par couplage d'hémistiches empruntés à des alexandrins bien connus ; tandis que, dépassant la simple juxtaposition propre au centon, ces programmes font éclore les joyeux et troublants sonnets de Rimbaudelaire, où le squelette morphosyntaxique du Dormeur du Val se trouve diversement « nourri » de vocabulaire baudelairien.

\subsubsection{Structures textuelles, génériques, de RDA}

Deux genres fictionnels classiques relèvent structurellement de la RDA : le roman épistolaire et les récits enchâssés.

Pour le premier, l'auteur y prend - sans dessein aucun d'être cru - le masque d' " inventeur », au sens archéologique du terme, d'une correspondance qu'il a eu à cœur de " publier $»^{33}$, et c'est à ce titre de " transmetteur » d'écrits d'autrui qu'il s'exprime ostensiblement dans des préfaces - cultivant l'ambiguité et le flottement ${ }^{34} \ldots$ - et éventuellement les notes dont il juge bon d'accompagner les lettres. C'est ainsi le texte dans son ensemble qui tient de la RDA : l'auteur - Rousseau, Laclos... - y occupe la place de $\mathbf{L}$, la forme unique mise en œuvre $\left(\mathrm{V}_{6}\right)$ est celle d'un DD, donné comme reproduction littérale des écrits d'une pluralité $\left(\mathrm{V}_{2}\right)$ de $\boldsymbol{l}$, précisément identifiés $\left(\mathrm{V}_{3}\right)$. Que celui qui « recueille et publie » la correspondance (Rousseau) ou s'est trouvé « chargé de la mettre en ordre » et de "l'élaguer » (Laclos) se manifeste plus ou moins au fil du texte - Rousseau annote plus que Laclos - la fiction de RDA d'un $\mathbf{L}$ s'adressant à nous, lecteurs, ainsi mis en place de $\mathbf{R}$, suffit à différencier le dispositif énonciatif - de RDA - de ces romans comme Le Lys dans la vallée ou les Mémoires de deux jeunes mariés, constitués également par une correspondance, mais à laquelle - hormis le nom de Balzac sur la couverture - nous avons fictivement accès, directement, sans intercesseur ayant choisi de nous la « rapporter ».

\section{2 www.alamo.free.fr}

33 Cf. La Nouvelle Héloïse, présentée comme « Lettres de deux amants - habitants d'une petite ville du pied des Alpes. Recueillies et publiées par J.-J. Rousseau ».

34 Prenant pour Les Liaisons dangereuses la double forme d'un « Avertissement de l'Éditeur » communiquant ses doutes sur « l'authenticité de ce recueil » et « ses fortes raisons de penser que ce n'est qu'un roman », suivi d'une « Préface du rédacteur » jouant à faire alterner sur deux pages le terme « Recueil » et celui d'« Ouvrage». 
Pour le second, c'est celui qu'illustrent ${ }^{35}$ Manon Lescaut, par exemple, ou La Petite Fadette. Le récit, fait à la première personne, par Des Grieux de son aventure avec Manon, comme celui du " chanvreur » contant un épisode de la vie berrichonne, sont explicitement donnés comme transmis à nous, lecteurs, par ceux qui les ont reçus : "L'Homme de qualité » qui rapporte dans ses Mémoires les circonstances de sa rencontre avec Des Grieux et la façon dont il a consigné le récit de celui-ci :

Je dois avertir ici le lecteur que j'écrivis son histoire presque aussitôt après l'avoir entendue, et qu'on peut s'assurer, par conséquent, que rien n'est plus exact et plus fidèle que cette narration. Je dis fidèle jusque dans la relation des réflexions et des sentiments que le jeune aventurier exprimait de la meilleure grâce du monde.

Voici donc son récit, auquel je ne mêlerai, jusqu’à la fin, rien qui ne soit de lui.

- J'avais dix-sept ans, et j'achevais mes études [...] (Manon Lescaut, $1^{\text {ère }}$ partie).

et George Sand qui, réfugiée à Nohant avec quelques amis lors de la révolution de 1848, et cherchant à « oublier tout cela, ne fût-ce que pendant une soirée ", fait appel au conteur de son village :

Le chanvreur ayant bien soupé, et voyant à sa droite un grand pichet de vin blanc, à sa gauche un pot de tabac pour charger sa pipe à discrétion toute la soirée, nous raconta l'histoire suivante ${ }^{36}$.

La forme textuelle est, ici encore, celle d'une RDA globale, au DD. Mais, contrairement au récit épistolaire où, orchestrant des voix diverses, parfois accompagnées de commentaires de "l'éditeur » des lettres, l'auteur ne se pose ici comme $\mathbf{L}$ que dans ce qui - préface ou introduction - fait office de "syntagme introducteur » pour ne plus intervenir ${ }^{37}$ dans le propos du narrateur, $\boldsymbol{l}$, unique $\left(\mathrm{V}_{2}\right)$.

Remarque 3 : Textes tout entiers au DD - par choix singulier. Ne relevant pas des genres évoqués ci-dessus, des textes, isolés, répondent aux choix - caprice, jeu, défi, expérience - d'un scripteur de raconter une histoire, faire le portrait d'une personne, évoquer une période... via le seul montage, collage brut d'énoncés reproduits au DD, voire en fac simile : outre le Portrait d'une Dame (cité chap. 5 (75), p 184) fait tout entier de bribes de paroles tombées de la bouche du

35 Pour m'en tenir à la simplicité des structures à deux étages, relativement aux cascades de récits des Mille et une Nuits, par exemple.

36 Fin de la préface, signée George Sand, de septembre 1848, suivie du chapitre 1 donnant, jusqu'à la fin du livre, la parole (soigneusement émaillée d'expressions berrichonnes) au chanvreur.

37 Non sans que, dans Manon Lescaut, le dispositif énonciatif d'un $\mathbf{L}_{\mathbf{i}}$ rapportant les propos $\mathrm{du} \boldsymbol{l}$ dont il a été le $\mathbf{r}_{\mathbf{i}}$ ne soit rappelé, en quelques lignes, entre la première et la seconde partie. 
« modèle », c'est le « Portrait d'une année » que vise Ph. Artières dans Miettes. Éléments pour une histoire infra-ordinaire de l'année $1980^{38}$, en composant une mosaïque de petites annonces parues dans le quotidien Libération; comme A. Camilieri ${ }^{39}$ entend restituer les péripéties d'une intrigue policière par le simple assemblage d'un « dossier » hétéroclite reproduisant exactement, en quasi fac simile, affiches, procès verbaux, lettres, rapports... relatifs aux événements.

\subsection{Discours vierges de RDA - « sans bordure »}

Il y a beaucoup moins de manières pour un Discours d'apparaître comme auto-suffisant, sans rapport avec des voix autres, qu'il n'y a de modes pour un Discours, comme on vient de le voir, de se faire tout entier support de discours autres.

$\mathrm{Au}$-delà du monologisme strict ${ }^{40}$ de l'auto-engendrement d'un discours mathématique à partir de ses axiomes, ou de l'imposition d'une vérité idéologique sourde à toute altérité, s'étend un territoire de discours " fonctionnels, notamment transmetteurs de savoir-faire ${ }^{41}$ (modes d'emploi, recettes, notices techniques, protocoles de manipulation, topo-guides), de savoirs avérés (manuels scolaires, par exemple $\mathrm{e}^{42}$ ), de croyances ou de principes indiscutés (catéchismes, articles de loi...) qui, tendanciellement se donne sans « bordure-interface ».

Remarque 4 : La RDA en bordure matérielle de Discours monologique. La Déclaration des Droits de l'Homme (texte de 1948) en offre un exemple. Les 30 articles qui la composent comportent (en faible quantité) deux types de référence à des actes de langage :

a) sur un mode abstrait, général, non actualisé comme un discours autre, tel

toute personne accusée d'un acte délictueux [...]. Nul ne sera condamné pour [...]. La volonté du peuple [...] doit s'exprimer par les élections [...].

b) sur le mode réflexif (outre le « Proclame » performatif initial) de la référence interne au texte lui-même, au dernier article, excluant qu' " Aucune disposition de la présente Déclaration » puisse être interprétée comme impliquant un acte contraire aux « droits et libertés qui y sont énoncés ». C'est dans le Préambule que cette Déclaration - relevant d'une énonciation « une » - se dote d'un ailleurs-avant de RDA qui la légitime et l'inscrit dans l'histoire :

38 Verticales-Gallimard, Paris, 2016, 139 p.

39 La disparition de Judas (La scomparsa di Patò, Mondadori Editore, 2000, traduction française par Serge Quadruppani, Métailié, Paris, 2002).

40 Cf. ci-dessus, p. 485, 422.

41 Dont Adam (1992) propose et analyse de nombreux exemples sous la rubrique « Discours procéduraux ».

42 Par opposition au discours de production ou de vulgarisation de savoir, $c f$. ci-dessous, p. 595. 
Considérant que [...] / - l'avènement d'un monde [...] a été proclamé [...] / - [...] les peuples des Nations unies ont proclamé leur foi dans [...] et qu'ils se sont déclarés résolus [...] / les États Membres se sont engagés à [...] / L’Assemblée générale / Proclame la présente Déclaration [...].

Cette observation - faite sur le mode d'une « rematérialisation » de la métaphore de la RDA envisagée comme "bordure-interface » de tout Discours - rejoint l'analyse des discours institutionnels menée par A. Krieg-Planque (2012 : 59 sq., 190) montrant, précisément, comment la légitimation des énoncés performatifs - dont ils sont producteurs attitrés - passe par le rappel préalable, sous le nom juridique de "considérants ${ }^{43}$, du réseau serré des textes et discours sur lesquels "s'appuient » résolutions et arrêtés d'instances... internationales ou municipales.

Dans ce modèle de discours, la bordure-interface de RDA se tient au seuil du Discours - occupant, comme « associée » $\left(\mathrm{V}_{4}\right)$, une place et remplissant une fonction d'entrée dans celui-ci.

\subsection{Entre les deux extrêmes du tout/sans bordure : pertinence de la variation}

On se contentera de noter, à travers quelques exemples, la pertinence de ce paramètre de l'Étendue $\left(\mathrm{V}_{1}\right)$ dans un Discours de sa $\mathrm{RDA}$, au plan générique $(a)$ puis à celui $(b)$ de la singularité des sujets parlants - non sans avoir, préalablement, souligné la différence entre un Discours, si saturé qu'il soit de formes de RDA renvoyant au discours dont il parle, et un Discours se donnant comme « tenant-lieu » d'un autre discours ${ }^{44}$.

De ce point de vue, la présence de la RDA dans les rubriques de presse consacrées à la vie des tribunaux ${ }^{45}$ diffère, autant par sa forme - quantité moindre, formes variées de RDA, présence de notations « didascaliques », appréciations personnelles du scripteur... - que par sa fonction (représenter et non « valoir pour »), des procès verbaux judiciaires visant la restitution neutre, intégrale et exclusive de l'échange verbal. De la même façon, parmi les genres de presse, il convient de nettement distinguer celui du « Portrait », issu d'un entretien entre le journaliste et la personnalité - si large qu'y soit le renvoi aux propos de celle-ci ${ }^{46}-\mathrm{du}$ genre, normé comme tenant lieu, de « l'entretien ». Notons encore qu'aucun genre rele-

43 Dont la formulation fait liste : « Vu l'arrêté du... ; Vu le code... ; Prenant note des rapports... ; Agissant en vertu du Chapitre de... » (cités in Krieg-Planque 2012 : 60-61).

44 Fonction dont on a vu qu'elle pouvait être remplie, par exemple dans le cas du « résumé », en l'absence de formes locales de RDA.

45 Cf. note 11 ci-dessus, p. 583.

46 Tels ceux qui figurent en dernière page du journal Libération, $c f$., pour ce genre du portrait, Mahrer et Tuomarla (2007), Da Cunha et al. (2018). 
vant de la critique littéraire, au sens large $\mathrm{e}^{47}$, faisant « contractuellement » place, par de la RDA, à son objet textuel, n'entend, si grande que soit cette place, « tenir lieu » du texte en question ${ }^{48}$.

\subsubsection{Pertinence générique des variations de l'Étendue $\left(V_{1}\right)$}

Deux domaines, très étudiés, donnent clairement à voir comment le degré $\left(\mathrm{V}_{1}\right)$ - et aussi la forme $\left(\mathrm{V}_{6}\right)$ - de présence de la RDA dans un texte est un élément de différenciation des genres, faisant partie du " savoir écrire » de ceux qui les pratiquent : presse écrite et production-transmission (écrite là encore) de connaissances.

Dans la presse se dégagent ${ }^{49}$ ainsi deux groupes à forte $v s$ faible étendue donnée à la RDA, incluant, pour le premier :

- les rubriques d'échos, potins, telle la page $2 \mathrm{du}$ Canard enchaîné dont la revue de la semaine politique passe, sous le titre « La Mare aux Canards » par une succession de paragraphes comportant chacun au moins un passage en DD, très marqué, cependant que la section « minimares » aligne sur deux colonnes des mini textes le plus souvent réduits à une seule phrase ;

- les articles d'information, d'analyses politiques, faisant état de débats, désaccords, etc. qui sont souvent tissés de RDA ;

et pour le second :

- l'éditorial qui, exprimant un point de vue personnel dans un texte assez court, fait rarement place à des formes marquées, explicitées, de $\mathrm{RDA}^{50}$, se donnant, note F. Claquin (1993 : 67), comme parole singulière destinée justement à être citée dans « les revues de presse [...] des radios dans les émissions du matin ».

Dans le domaine des discours " de la connaissance ", la distinction nettement établie entre ce qui relève de la production de connaissance et des deux types de

47 Ouvrages universitaires ou " grand public », rubriques livres de journaux et et magazines, " émissions littéraires », exercices académiques d'explication ou de commentaire de textes, discours de réception à l'Académie française...

48 Comme y vise, par exemple, une traduction.

49 Cf. notamment « Genres de la presse écrite et analyse de discours » Semen 13 (2001), Adam (1997), Moirand (2007) - offrant un panorama des marquages morphosyntaxiques, associés systématiquement au doublon guillemets et italiques.

50 Conformément, note S. Moirand (2001), à son appartenance à la catégorie qu'elle pose des « genres à énonciation subjectivisée » (vs « objectivisée »). 
discours second par rapport au premier que sont la vulgarisation scientifique et la transmission « académique » des savoirs, se reflète dans leur bordure de RDA, au plan des variables $\left(\mathrm{V}_{1}\right)$ de l'Étendue et $\left(\mathrm{V}_{3}\right)$ de la Spécification des discours autres.

Le caractère "multiréférentiel $\|^{51} \mathrm{du}$ discours scientifique ${ }^{52}-$ passant de surcroît par des « adresses interdiscursives » comportant noms propres d'auteurs, de travaux, indications précises de localisation - a été largement mis en évidence comme manifestation de son mode de progression foncièrement dialogique et, solidairement, de son positionnement - accord, conflit, filiation, légitimation dans le champ discursif concerné (émanant de pairs et d' « autorités »). Considéré comme trait caractéristique du discours scientifique ${ }^{53}$, la maîtrise de ce type de bordure - importante et à autres spécifiés - est envisagée comme relevant d'un "savoir faire citationnel spécifique », demandant un apprentissage ${ }^{54}$.

Le discours de vulgarisation scientifique, lui aussi très étudié ${ }^{55}$, fait appel ostensiblement aux discours autres des « savants-spécialistes-experts » dont il s’affiche - «troisième homme » chargé de rétablir le lien rompu entre la Science et la Société... - comme le médiateur en direction du grand public. La densité de RDA y est aussi forte $\left(\mathrm{V}_{1}\right)^{56}$ qu'est faible la Spécification $\left(\mathrm{V}_{3}\right)$ des ailleurs convoqués, la production d'une image ou d'une « mise en scène » de la transmission de connaissance s'imposant nettement dans ce discours, à côté, ou à la place, de l'activité, déclarée, de transmission... ${ }^{57}$

S’opposant aux deux genres précédents, ce qui relève d'une visée didactique - manuels scolaires ou universitaires, encyclopédies... - tend à l'effacement ${ }^{58}$ de l'origine du savoir énoncé et a fortiori de la pluralité, éventuellement discordante, des sources, se rapprochant dans cette énonciation de vérités établies des discours monologiques « sans bordure $»^{59}$.

51 Grossman (2011 : 208).

$52 C f$., chap. 5.3.2, p. 162, références et « illustration » proposées par Perec.

53 Simonin-Grumbach (1975).

54 Boch et Grossmann (2001) notamment.

55 Cf. Mortureux (dir.) (1982), Moirand (2001), par exemple.

56 « patchwork de dires tenus ailleurs » dit Moirand (2007).

57 Sur le mode, multiplement analysé par Goffman (1959/1975), où « la mise en scène de la vie quotidienne » peut « transformer une activité en spectacle» (p. 40).

58 À titre d'exemple, une étude (Plazaola et al., 1995) met en évidence, sur un important corpus d'« économie ", " la polyphonie restreinte à l'œuvre dans les manuels et encyclopédies par rapport à la presse quotidienne accueillant une « diversité de voix ».

59 Constat à ne pas étendre à la transmission orale, interactive, des cours magistraux dont Claudel (2011) décrit les « pratiques citationnelles », notamment via des DD et MAE lus à haute voix. 
Dans « Une Histoire de discours » S. Moirand (1986), étudiant de façon pionnière l'important corpus de 20 années de la revue pédagogique Le Français dans le Monde, fait, dans la mise au jour de la dualité de discours qu'elle y fait apparaître, une place cruciale à la « bordure » de RDA qu'ils se donnent, comme témoignant de la « représentation qu'ils ont d'eux-mêmes et de la place qu'ils occupent dans le champ $»^{60}$ : l'un se présentant comme homogène, monologique, transmettant des « savoir-faire » pour une classe de langue, l'autre, qui diffusant des savoirs issus des « champs connexes » (la linguistique et ses théories, notamment) « avec lesquels il flirte et dont il se démarque », " exhibe son hétérogénéité » ${ }^{61}$ sur un mode qui «flirte » aussi avec la vulgarisation.

\subsubsection{Pertinence individuelle de la variation en Étendue $\left(V_{1}\right)$}

Je ne tenterai pas d'esquisser un dégradé allant de Hérille, Montaigne, Flaubert... ou tel sujet doublant son dire d'une basse continue de comme on dit, à ce qu'on dit, on dit, etc., jusqu'à des sujets qui, de façon singulière, font le choix - délibéré ou insu - de s'énoncer sur un mode solitaire, altièrement dépourvu d'appuis et de garants, élégamment « désencombré » de ses échafaudages, ou allégé de ses dettes... On retrouvera plus loin cette variation d'amplitude de la bordure du dire à travers la façon dont elle est articulée aux autres variables.

Cette dimension personnelle est évidemment à l'œuvre dans tous les genres, dès lors que leur « norme de bordure » n'est pas strictement contraignante : on en évoquera seulement quelques manifestations.

Ainsi, dans leur variété, les multiples discours ayant pour objet déclaré, fictif ou réel, un sujet humain - mémoires, souvenirs, (auto)biographies, récits de vie, récits de cure (ou de « cas ») psychanalytique - font tous place à la parole de celui dont ils parlent, mais cela en quantité et selon des formes très variées.

La cure psychanalytique étant " une histoire de parole », son " écriture » - difficile - emprunte quant à l'étendue donnée à la RDA (et aux formes qu'elle prend) des chemins différents à l'extrême ${ }^{62}$, depuis l'expérience - problématique - de la pure et intégrale restitution au DD des propos tenus ${ }^{63}$, jusqu'au retour d'un analyste, à des fins théoriques, sur le déroulement d'une cure

60 Moirand (1986: 27).

61 Ibid. : 32.

62 Question à laquelle s'est attachée un groupe de travail (2006-2009) auquel renvoie F. Sitri sur un corpus de 7 textes $(2012: 263-273)$.

63 Choix radical qui semble s'ébaucher dans le « dispositif théâtral », analysé par Sitri (2012) dans un texte de D. Vasse de 1995 figurant dans le corpus étudié. 
dont le récit « passe », de façon bien moindre, par une diversité de formes de $\mathrm{RDA}^{64}$.

Sur le versant « attaché à l'objet » de la variable $\left(\mathrm{V}_{4}\right)$, les genres et domaines à « objet humain » - récit de voyage en terre étrangère, étude sociologique, ethnologique « de terrain », évocation du passé ou écriture de l'histoire - présentent un fort gradient de différence dans la place faite au discours « autre » par le lieu, le milieu, le temps...

Ayant évoqué ci-dessus la norme d’avancée « multiréférentielle » du discours de recherche scientifique, on peut noter les écarts importants qu'y inscrivent les singularités d'écriture : la mise en regard de deux importants recueils de textes de linguistique, de Benveniste et Culioli - les tomes 1 des Problèmes de linguistique générale (1966) et de Pour une linguistique de l'énonciation (1990) - fait apparaître immédiatement - ce qu'un examen attentif confirme - une différence saillante quant à l'étendue de leur «bordure » de RDA. Au fil des notes de bas de page référant de façon précise, chez Benveniste, à d'autres travaux, l'avancée du discours se fait dans et en rapport avec une importante " société » de linguistes ${ }^{65}$, là où frappe l'extrême « minceur » de l'interface avec l'ailleurs des autres discours dans l'écriture scientifique, tendanciellement « solitaire » de Culioli ${ }^{66}$.

\subsubsection{Stéréotypie réprobatrice à l'encontre du « trop de citations »}

Il faut noter que - hormis les appréciations, de type académique, sur le caractère bien ou insuffisamment « informé » d'une étude, ou l'éventail des réceptions à des « exercices de type citationnel » comme ceux de Perec par exemple - la tonalité des remarques sur les bordures-interfaces importantes est, de façon dominante, péjorative.

Si c'est comme « constellée de citations » que l'écriture (d'assemblages et collages) de Mauriac - «petite planète » aspirant à « se trouver » dans « la Voie

64 Cf. par exemple le texte de Pontalis, de 1995, figurant dans ce même corpus.

65 Les 10 articles constituant les parties III et IV de Benveniste (1966 : 91-222) présentent nettement plus d'une référence en note par page (177 sur 121 pages, sauf erreur...) convoquant Saussure, bien sûr, mais aussi Meillet, Vendryès, Bloomfield, Sapir, Troubetskoï, Hjelmslev, Seebok, Godel, Malmberg, Guillaume, Marouzeau, Tesnière, Damourette et Pichon, Gougenheim, etc. : un panorama...

66 En tenant compte de la bibliographie de 11 titres jointe (p. 80) à un article et d'un renvoi « large » (ce que X, Y, Z etc. appellent..., p. 51), le taux moyen de référenciation à un ailleurs discursif est de une référence par dizaine de pages (10+11 sur 205 pages, les échanges interlocutifs lors de discussion d'une présentation orale préalable de quelques articles, reproduits en fin d'articles, relevant d'une autre logique). 
Lactée » de ses admirations - peut être évoquée ${ }^{67}$, il n'en va pas de même lorsque Maurois caractérise la prose de Péguy comme " hérissée de citations ${ }^{68}$ ou que dans quelque chronique littéraire est dénoncée chez un auteur ${ }^{69}$ « la manie de nous abreuver de citations en sautant d'un sujet à l'autre »...

« Manie, travers » - bêtise même lorsque l'ailleurs convoqué est celui de la sagesse des nations, le recours fréquent aux dires autres est aisément jugé abusif, taxé de psittacisme, témoignant d'un défaut de pensée-parole propre ${ }^{70}$.

Ainsi, toute « bordure » (ou « peau ») du discours, génériquement non-contrainte, dit quelque chose de son énonciateur, de sa manière propre d' «être-avec » les discours autres - et les autres -, partie prenante de sa « manière d'être » au monde et au langage. Sous le titre « Le regard de Narcisse » une étude consacrée à Théophile Gautier, articulant « l'homme » à l'œuvre et à son style..., met en regard « une structure psychique originale » et des traits marquants de son écriture, dont un recours «maniaque » à la citation. Décrivant Gautier comme « détaché de la vie », souffrant d'une « absence », « inadhérence » au monde et au réel, M.-C. Schapira (1984 : 106 sq.) perçoit chez lui « une démarche défensive » contre le «vacillement du réel » et du « contour » empruntant deux voies : une recherche de la « matérialité des formes » ${ }^{71}$ par la minutie hyper-réaliste et érudite de ses descriptions et, partie prenante de l'édification d'un " rempart de mots, de références » assurant des « conditions de clôture de sécurité », une « habitude de la citation » - si puissante que, «fétiche, efficace », elle se fait « tic de plume » s'offrant aux décomptes... ${ }^{72}$

67 F. Gai (2011), $c f$. ci-dessus chap. 12.2.3.2, p. 486.

68 Maurois, Études littéraires, t. 1 : 235-236.

69 G. Martin-Chauffier, à propos d'un ouvrage de Ch. Dantzig, Paris Match, 10-16 sept. 2015.

70 Analyse à tempérer... dès lors qu'on se retourne vers les cas (cf. chap. 13) de vraie défaillance de parole propre, où, précisément, c'est une parole « comme citée » que produisent - je ne dis pas « énoncent »-des sujets qui ne sont pas en mesure de « citer », c'est-à-dire de " dissimiler » le dedans de la parole propre, du dehors du langage.

71 Cf. cette remarque de Gautier lui-même dans Mademoiselle de Maupin : "Aussi, par une espèce de réaction instinctive, je me suis toujours désespérément cramponné à la matière, à la silhouette extérieure des choses [...]. »

72 L'auteur faisant état dans Les Jeunes-France de 313 « citations de noms d'auteurs et d'œuvres », 308 dans le recueil des Nouvelles, 314 dans les Romans et Contes... (soit une par page environ), ce qui - même si le sens de « citation » n'est pas précisément spécifié - devrait correspondre à une consistante « bordure». 


\section{Variable $\left(\mathrm{V}_{2}\right)$ : Taux de Dispersion des discours autres}

Cette variable vise la diversité des $\boldsymbol{l}$ représentés dans le Discours : on ira de la concentration de l'ailleurs du Discours sur une source unique jusqu'à sa dispersion en une multiplicité de sources distinctes, en observant quelques configurations types - pluralités fortement structurées ou association d'un centre et d'une marge en halo dispersé. Cette variation se manifeste aussi bien entre les genres, les domaines, les styles personnels : on ne se propose que d'en donner quelques exemples.

Elle ne se confond pas avec le nombre d'actes $\boldsymbol{a}$ distincts représentés : un recueil des bons mots de Sacha Guitry tout au long de sa vie est un discours à ailleurs unique - à taux de Dispersion $\left(\mathrm{V}_{2}\right)$ nul. Elle ne se confond pas non plus avec le nombre de sujets parlants, leur identité singulière ou collective : c'est l'hétérogénéité des sources convoquées dans la bordure qui est en cause ; l'ailleurs dont un Discours se borde est aussi peu « dispersé » si c'est au « on » d'un groupe, milieu... qu'il réfère ou à « la totalité les études sur le sujet » ${ }^{73}$, qu'à « mon cousin qui est très bien informé » ou au « Président Mao ».

Il s'agit, si l'on veut, pour la bordure, interface du Discours et de son extériorité, lieu par définition hétérogène, de sa « texture », de son " grain » plus ou moins hétérogène selon la diversité des ailleurs qui y jouent.

\subsection{Centration du Discours sur un extérieur unique.}

Exclusive ou seulement très dominante, la centration sur un discours autre opère également suivant les deux modes d'Émergence $\left(\mathrm{V}_{4}\right)$ du discours autre comme (2.1.1) « attaché à l'objet » ou (2.1.2) « associé » à sa progression.

\subsubsection{Ailleurs unique attaché à l'objet $\left(\mathrm{V}_{4}\right)$ du Discours}

Le fait pour un Discours de se donner une bordure-interface « attachée » $\left(\mathrm{V}_{4}\right)$ - sans distraction aucune ou presque - au discours autre unique dont il a fait son objet, peut relever de plusieurs fonctionnements discursifs :

- celui de l'espace générique du « tenant lieu » ( $c f$. ci-dessus) où la moindre référence de la part du rédacteur à quelque autre discours autre serait tout à fait incongrue :

73 Ou encore à la houle des paroles sur la place Saint-Marc ou à l'écoulement verbal des « tricoteuses », $c f$. chap. 5, exemples (73) et (74), p. 183. 
?? Compte rendu - puisque c'est comme cela qu'on l'appelle maintenant - de séance de la commission

- celui (opposant la vive implication personnelle de leur énonciateur à la neutralité «professionnelle » des rédacteurs de « tenant-lieu») des Discours polémiques qui présentent volontiers cette centration exclusive sur le discours visé ; celle-ci, qui n'est pas génériquement contrainte ${ }^{74}$, manifeste plutôt une focalisation passionnelle sur l'ennemi à abattre, affronté, solitairement, sans soutiens extérieurs... Il en va ainsi, parmi des Discours étudiés précédemment ${ }^{75}$ pour la place démesurée qu'ils font - via la MAE - aux mots ennemis, et qui, au-delà de cette forme, font « flèche de tout bois » en matière de RDA, de la très guerrière « Lettre Ouverte » de la psychanalyste L. Irigaray adressée à « Messieurs les psychanalystes », visés en vous comme cible unique - d'autant plus « unique » que se trouve explicitement dénoncée l'abolition de la singularité des personnes réduites à une « meute » indifférenciée de porteurs de la parole du Père-Maître - déployant densément, dans l'attaque d'un seul autre, toutes les ressources formelles de la RDA ;

- celui, aussi, parmi les genres de discours à large bordure de RDA évoqués ci-dessus (1.3) ceux, nombreux, dont la RDA renvoie exclusivement ou quasi-exclusivement au discours tenu dans l'ouvrage ou par la personne dont ils parlent (recension, critique littéraire, portrait...), auxquels on peut joindre dans les biographies la prédominance des propos de ceux qui en sont l'objet $^{76}$, ce que la lecture des Évangiles, comme recueil des dires de Jésus, ne vient pas contredire ;

74 Ainsi le pamphlet anti-lacanien de F. Georges L'Effet 'yau de poêle de Lacan et des lacaniens, Paris, Hachette, 1979, « visant » sa cible principale, est-il assez librement ouvert à d'autres ailleurs... (cf. Authier-Revuz, 1995/2012: 335).

75 Dans Authier-Revuz (1995/2012 : 333-335) la diatribe Assez décodé lancée en 1978 par R. Pommier contre « la nouvelle critique » qui ne convoque nommément quelques auteurs - via diverses formes de RDA que comme autant de pantins, quasi-interchangeables, de la comédie intellectuelle en cours faite d' "imposture », de " cuistrerie »; ou un texte (Mémoire en défense. Contre ceux qui m'accusent de falsifier l'histoire, La vieille taupe, Paris, 1980) - aussi odieux que « fou »de Faurisson dressé contre le «mensonge » du « discours exterminationiste » relatif au sort des juifs pendant la seconde guerre mondiale, qui inscrit le face à face avec un seul autre et dans un seul rapport, $\left(\mathrm{V}_{5}\right)$, « meurtrier ", à cet autre, mettant en jeu le " droit à l'existence pour l'un des deux discours seulement » (Authier et Romeu, $1984: 67$ ).

76 Associés aux propos d'énonciateurs - parents, amis, relations... - portant sur cette même personne. 
- celui, enfin, de textes littéraires qui font, de façon singulière, le choix d'un $\boldsymbol{l}$ omniprésent et unique (ou presque) : on a évoqué ${ }^{77}$ l'expérience d'écriture du long volume à un seul je du Portrait d'une dame d'A. Frontier. Deux textes d'Annie Ernaux ${ }^{78}$ présentent cette centration sur une altérité, individuelle pour l'un, « ma-mère-elle », collective pour l'autre : le « on » de la rumeur sociale qui fait le fond de Les Années. Du premier on peut dire qu'il est - au fil de chacune de leurs rencontres « depuis que [sa] mère a commencé de présenter des pertes de mémoire » - un « Journal des phrases » énoncées par celle-ci, tentative dépouillée et émouvante de sa fille de « recenser toutes ses phrases alors qu'elle ne parle presque plus » (p. 75), en témoin bouleversé mais soucieux de les recueillir exactement (quasi-exclusivement au DD) : au cœur de (presque) chaque brève séquence, datée du jour de la visite, une phrase qu'elle dit, raconte, ajoute... ou le souvenir de ce qu' " elle disait » autrefois (« cette phrase qu'elle disait », " souvent elle disait », " une de ses phrases... »), sa parole, «fixée », domine le texte : la diversité des autres voix - de ceux qui lui parlent, ou parlent/parlaient d'elle, de l'entourage anonyme, exceptionnellement émanant du monde extérieur (radio...), ne forment qu'un accompagnement ${ }^{79}$.

Au livre du «Elle dit » consacré à sa mère, répond, avec Les Années, celui du "On disait », " sorte d'autobiographie impersonnelle ${ }^{80}$, fondue dans la rumeur collective dont des séquences - parfois à partir de photos - évoquant ce qu'elle faisait, croyait, pensait..., ne rompent pas le cours, illustrant l'exergue de J. Ortega y Gasset : « Nous n'avons qu'une histoire et elle n'est pas à nous ».

"Récit glissant dans un imparfait continu, absolu » dit l'auteur ${ }^{81}$, ce texte n'accueille aucune parole d'autres singuliers ${ }^{82}$, aucun « je », mais ${ }^{83}$ la « parti-

77 Chap. 5.5.2.1 p. 184.

78 Je ne suis pas sortie de ma nuit, Gallimard, 1997, et Les Années, Gallimard, 2008.

79 Pour le journal des visites des années 1983-1985 (p. 15-52) c'est « elle » dont la parole est représentée dans $75 \%$ des formes de RDA.

80 Les Années p. 40 et « Elle ne regardera en elle-même que pour y retrouver le monde, la mémoire des jours passés du monde, saisir le changement des idées, des croyances et de la sensibilité [...]. » (p. 239)

81 Ibid, p. 240.

82 Sinon celles de voix «publiques » d'auteurs de chansons, de films, de livres, d'hommes politiques, de présentateurs d'émissions télévisées, dont les titres, les mots font partie du « fond sonore » du moment.

83 L'effacement du singulier se faisant ici plus en écho à la pensée du «social », comme principe de tout chez Bourdieu (comme le note L. Rosier, 2012 : 380 sq.), que dans le vertige de la dépossession de Flaubert et de ses «idées reçues ». 
tion » que le « langage construisait avec constance ${ }^{84}$ pour qu'elle soit reprise - en position de « sujet » de divers verbes de dire - principalement par l'incessante voix du « on », démultipliée en :

les gens, les parents, les enfants, les jeunes, les élèves, les riches, tout le monde, quelqu'un, nous, le leitmotiv, la conversation, la réclame, une pub, l'imagination commerciale, le discours du plaisir, etc.

qui

disait, racontait, annonçait, évaluait, affirmait, convenait, répétait...

des mots - énoncés au DD, en fragments autonymes ou en MAE.

\subsubsection{Ailleurs uniques - ou hyper-dominant - associé $\left(\mathrm{V}_{4}\right)$ au Discours}

On a rencontré les deux genres du pastiche et de la parodie qui relèvent intégralement de cette mise en jeu d'un autre discours non pas comme celui dont on parle mais de celui avec lequel, comme lequel, en symbiose avec lequel, on parle d'un référent quelconque (tel «l'affaire Lemoine » dont Proust parle avec/comme Balzac, Flaubert...).

Un autre mode, souvent observé, rarement revendiqué par les énonciateurs, qui en sont d'ailleurs très inégalement conscients, et volontiers, au contraire, dénoncé, raillé... par ceux qui le notent, est celui de l'association récurrente à un discours autre, dans un Discours où l'appel à cet autre - généralement au titre de garant, porteur de vérité... - peut atteindre au statut d'une sorte de « préfixe » à toute assertion...

En relèvent, au fil de l'histoire, soulignés de façon critique par des observateurs extérieurs au groupe que ces références contribuent à « cimenter », les

Aristote a dit..., Hippocrate ${ }^{85}$ dit..., Les Saintes Écritures le rappellent..., Il est dit dans le Petit Livre Rouge..., On trouve dans le Coran..., Selon Lacan...

à la fonction de signe de ralliement placé ostensiblement en tête de parole.

84 Les Années, p. 213.

85 Dont se gausse Molière dans Le Médecin malgré lui, II-2 : «Sganarelle, en robe de médecin, avec un chapeau des plus pointus : Hippocrate dit... que nous nous couvrions tous deux./Géronte : Hippocrate dit cela ? /Sg. : Oui./ G. : Dans quel chapitre, s’il vous plaît ? Sg. : Dans son chapitre... des chapeaux./ G. : Puisque Hippocrate le dit, il le faut faire. ». 


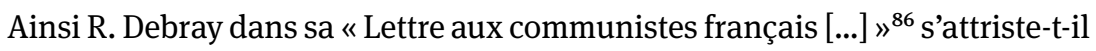
du fait que

Tout oppositionnel sait - enfance de l'art - qu'il lui faut se couvrir en citant d'abord à plusieurs reprises les propos du secrétaire général. « Comme le disait Georges Marchais dans sa dernière intervention... » - sans ce blanc-seing pas de salut, pas d'écoute. Rien de plus attristant que ce péage donnant le droit de circuler dans les avenues du Parti [...]. (p. 118)

De même que, dans le quotidien Libération $^{87}$, un lecteur ironisant sur le «Comme l'a si bien dit le Président Sarkozy (ou comme il le dira) » qui règle le discours de ses partisans, en recueille, sous le titre «Sarkolâtries », les « variations » dans une tribune publiée dans le journal :

Le temps est venu en effet, ainsi que l’a évoqué Nicolas Sarkozy [...] Très précisément, Le Président a exprimé son désaccord [...] À cet égard, le Président a montré le chemin [...] et Faisons nôtre l'indication de Nicolas Sarkozy pour [...]. (idt)

Ce garant exclusif appelé en protection initiale de sa parole, qui n'est évidemment pas limité au champ idéologico-politique, apparaît communément dans la vie quotidienne : enfant traversant des périodes de « mon frère m’a dit... », épouse soumise se retranchant derrière un " mon mari pense que... », un des fils de F. Mitterand, ironiquement surnommé « papa-m'a-dit » dans le peu bienveillant petit monde politique, ou encore, inattendue pour qui songe au caractère affirmé - voire péremptoire - de sa parole ultérieure, cette évocation, par sa biographe, de S. de Beauvoir, jeune fille

[qui] elle-même avait toujours à la bouche le nom de Maheu et ne pouvait commencer une phrase sans dire d'abord ce que lui pensait en la matière ${ }^{88}$.

\subsection{Pluralité structurante d'extérieurs}

C’est le cas que présentent, par exemple, des textes théoriques visant à prendre position, relativement à une question en débat dans un champ qu'ils représentent comme traversé et configuré par plusieurs courants : je renvoie à l'analyse, faite de

86 1978, Seuil, Paris.

87 25-07-2007, Courrier des lecteurs.

88 D. Bair, Simone de Beauvoir, traduction française, 1991, Fayard, Paris, p. 148. 
ce point de vue, de deux articles ${ }^{89}$ dans lesquels le fonctionnement observé pour la MAE vaut pour l'ensemble de la RDA.

On voit ainsi dans un texte dont l'objet - ce dont il parle - est un article de Bourdieu (A), J.-L. Houdebine ${ }^{90}$ faire appel à deux extérieurs d'appui : (B) Benveniste et (C) Freud-Lacan pour soutenir sa critique du premier (A).

C'est en passant par une configuration plus complexe que P. Encrevé - visant, dans sa présentation d'un numéro de revue intitulé « Linguistique et socio-linguistique », à penser la seconde comme partie légitime de la première - se positionne, à travers un réseau serré de rapports divers, dans une pluralité structurée de six extérieurs représentés : établissant d'abord des rapports de filiation à la dialectologie américaine (A), d'appui à la sociologie de Bourdieu (B), d'opposition à la grammaire chomskienne (C) ; pour, secondairement, revendiquer l'héritage saussurien (D), ceci conduisant à prendre ses distances vis à vis d'une « vulgate » du discours linguistique en matière de variété (E) et à critiquer le discours d'autres sociolinguistes $(\mathrm{F})$.

Loin des rapports d'opposition/alliance/filiation structurant un champ scientifique, P. Von Münchow souligne, dans son étude contrastive des « guides parentaux » français et allemands ${ }^{91}$, que ce discours se déroule en passant par «Quatre locuteurs rapportés principaux [...] : les experts, la doxa (et/ou l'entourage de la famille), l'enfant et le parent » selon des « distributions » de la parole quantité, formes de RDA... qui en éclairent les fonctionnements. Le point que je retiens ici est que cette RDA, quadripartite, " fait société » de ces divers autres, interdépendants, instituant cette configuration d'autres parlants comme un trait - sujet à variation - du genre du « conseil éducatif ».

\subsection{Dispersion des autres dans la bordure}

Au-delà des pluralités organisées « d'autres », s'étend le domaine des autres disparates, éparpillés sans hiérarchie. Positionnement idéologique, stratégie politique, disposition psychique singulière, trait d'écriture... sont susceptibles de passer par cette dispersion des autres convoqués, dont on peut observer que - sans s'y confondre - elle présente une forte affinité avec le versant " associé » du mode d'émergence $\left(\mathrm{V}_{4}\right)$ dans le Discours.

89 In Authier-Revuz (1995/2012 : 429-431).

90 J.-L. Houdebine (1976a).

91 Von Münchow (2011) et (2012). 
Notons que, génériquement « dispersés », les genres « collecteurs » de discours dispersés - florilège de citations relatives à un thème ou dictionnaire de citations associées à un mot - relèvent, genres limites du point de vue de cette opposition $\left(\mathrm{V}_{2}\right)$, d'une dispersion « de principe », organisée autour d'un thème, de chacun des mots.

Dans le champ idéologique, l'étude que D. Maingueneau ${ }^{92}$ a consacré à l'opposition qui traverse le champ du discours religieux au XVII ${ }^{\text {ème }}$ siècle en France, entre l' " humanisme dévot » des jésuites et le mouvement janséniste, fait exemplairement apparaître cette variable $\left(\mathrm{V}_{2}\right)$ du taux de Dispersion comme un point saillant de leur divergence : l'étendue et la diversité de la « bordure-interface » par laquelle les premiers manifestent leur ouverture sur le monde profane et leur souci d'en concilier les multiples visages à la doctrine chrétienne dans une dispersion de leurs ailleurs, est clairement un trait auquel les seconds répondent par une centration rigoureuse sur le cœur, notamment primitif, du discours de l'Église, assortie à leur idéal de solitaires. Je renvoie particulièrement aux pages (p. 145-148) consacrées aux bibliothèques des uns et des autres, comme concrétisation de l' « espace du citable » qui, pour les jésuites,

contiendra les ouvrages des meilleurs auteurs de l'Antiquité gréco-latine, des traités de cosmologie, de rhétorique ou d'histoire naturelle... à côté des textes de la tradition et de l’Écriture.

là où la bibliothèque janséniste

cherche à coïncider le plus exactement possible avec le corpus de l'Église, posé comme un univers textuel restreint, clos, stable et homogène, dont la dispersion spatio-temporelle est conjurée par sa résorption dans la ponctualité d'un unique auteur, l'Esprit-Saint. (p. 146)

Le champ politique offre un bel exemple de stratégie offensive de renouvellement d'une pensée - celle de la droite des années 1970-1980 - par l'ouverture la plus dispersée qui soit à des ailleurs nouveaux et imprévus, rompant avec l'interface frileux de références usuelles aux garants ou ennemis reconnus : celui - étudié avec précision par S. Bonafous et P. Fiala ${ }^{93}$ de la revue Éléments, fer de lance d'une Nouvelle Droite dont les éditoriaux tranchent, par leur bordure « efferves-

92 Maingueneau (1984), chap. V, partie 2 notamment, dont on retrouve de précieuses présentations synthétiques dans (1987: 89) ou (1991 : 200-202).

93 Bonafous-Fiala (1986), Fiala (1986) dont Authier-Revuz (1995/2012 : 435) propose un résumé soulignant, dans l'esprit du parcours suivi dans le présent ouvrage, le « jeu » de variables que dégage leur précieuse étude. 
cente » - en quantité et diversité - non seulement avec le mode dominant (sobre en RDA) de l'écriture du genre de l'éditorial, mais aussi avec l'extériorité révérencieusement centrée, dans L'Appel, sur De Gaulle au titre de père fondateur, comme avec la focalisation marquée sur le discours de l' " adversaire de gauche » que partagent plusieurs publications de droite affirmée ou extrême ${ }^{94}$. Le numéro 1 de la revue Éléments, dans un éditorial ${ }^{95}$ dirigé contre les déclarations pacifistes de l'église de France, donne le ton, affichant une « bordure-interface » qui, à travers les formes les plus diverses de RDA, tend à recouvrir quasiment tout le texte, et dans laquelle se rencontrent ${ }^{96}$, amis ou ennemis ${ }^{97}$, non seulement :

personnalités civiles et surtout militaires, représentants de la hiérarchie de l'église de France, l’Église, les officiers, les évêques...

mais aussi

les « défavorisés », l'empire Romain, les citoyens de la Jérusalem céleste, Spengler et Tertullien (De l'idolâtrie XIX et De Corona XI), etc.

L'exemple d'une bordure, reflet tragique de la difficulté pour un sujet à « être avec » les autres sur un mode « vivable », est celui que présente le récit à la première personne, par une jeune fille, de son internement de quatre mois pour anorexie en hôpital psychiatrique ${ }^{98}$. Cri de refus d'un bout à l'autre, ce texte s’apparente aux textes intégralement polémiques évoqués ci-dessus ; mais ceuxci, passionnément attachés à leur cible, rejetaient un discours autre ; rien de tel ici pour un être qui, rejetant le monde qui l'entoure, rejette tous les mots des discours les plus divers - ceux des « ils » dont elle répète " ils ne m'auront pas », des « eux » du « on », parents, mère en particulier, médecins, soignants, discours

94 La Lettre de la Nation, Le National, Militant.

95 Par Alain de Benoist (alias Robert de Herte).

96 En tant qu'ils « parlent, disent écrivent, déclarent, répondent, ajoutent, donnent à choisir, demandent, affirment, polémiquent, critiquent, dénoncent, attentent au moral de l'armée ou provoquent [les] militaires à la désobéissance... ».

97 La stratégie consistant à faire appel aux mots de l'adversaire, pour s'en emparer en les « retournant », a été souvent mise au jour. Cf. par exemple : Constantin de Chanay (2010), dans son étude du débat (2007) entre S. Royal et N. Sarkozy, montrant comment dans le " profil idéologique » (p. 311) que chacun dessine de lui-même par sa " convocation sélective » de tiers extérieurs, il apparaît comme une spécificité du second « de ratisser plus large, de préférence à gauche, sur les terres de son adversaire [Mitterand, Zapatero...] « comme il l'avait fait en d'autres circonstances avec Jaurès. » (p. 317)

98 V. Valère, Le pavillon des enfants fous, Éd. Stock, Paris, 1978 ; cf. Authier-Revuz (1995/2012) : 422-424). 
social anonyme - sans aucune position de repli, c'est-à-dire dans l'omniprésence d'un discours extérieur honni, sans « intérieur » possible comme « lieu » propre où habiter. Le rejet des discours autres « vomis », " qui ne devraient pas exister » s'étend, bien au-delà du lexique médical (" "nourrir une névrose” comme ils disent », p. 220), au « vocabulaire de tout le monde » («famille, fils, mère, mettre un enfant au monde, gâcher, profiter de la vie »), et c'est l'ensemble de la RDA qui est à l'unisson de ce refus tous azimuts du monde et de tous les discours qui le disent - refus qui, tragiquement, cherche une issue - qui ne sera pas trouvée dans le fait de le dire :

Je suis seule [...] à vomir ces gens [...] cette société [...] Gardez-le votre monde [...] je ne l'accepterai jamais, ce n'est pas le vrai [...] Je ne veux pas dire les mêmes mots [...] je ne veux pas utiliser le même langage, je ne suis pas de leur monde, je préfère me tuer. (p. 15, 19, 43)

Bien entendu, la dispersion des autres dans une dense interface revêt d'autres couleurs que celle de cette asphyxiante bordure « de combat » contre tous. Montaigne, Proust, Pérec, San Antonio... et tant d'autres sont là pour témoigner que la dispersion des autres " associés » $\left(\mathrm{V}_{4}\right)$ dont, diversement, s'accompagnent, « en bordure », des écritures littéraires, s’inscrit - riche, nourricière, joueuse, imprévisible, réactive, curieuse, gourmande ... - du côté d'un « être-avec » vivant.

\subsection{Forte centration et frange de liberté}

La combinaison dans la bordure d'un Discours entre un autre hyperdominant et des autres « dispersés » à émergence unique s'observe avec une régularité quasi-générique dans des Discours - écrits ou oraux " formels » - explicitement consacrés à un objet discursif ; l'opposition $\left(\mathrm{V}_{4}\right)$ attaché/associé venant redoubler le caractère centré/dispersé des extérieurs représentés : à la forte - voire massive - présence, contractuelle pourrait-on dire, de l'autre dont le Discours s'est chargé de parler, répondent les appels ponctuels à des voix autres, diverses, choisies librement par $\mathbf{L}$, comme « associées », pour accompagner son propos.

D’un tel « schéma de bordure » relèvent des genres aussi différents - canal, étendue, contexte... - que (a) des monographies consacrées à un auteur ou (b) le genre rituel des discours de réception à l’Académie Française.

a) La précieuse traversée de l'œuvre de Balzac au prisme de « La Vie quotidienne dans La Comédie humaine », dans laquelle Ph. Berthier (1998) guide le lecteur, s'en remet pour une large part à la parole de Balzac : encadré par l'annonce en 
page de garde : «Toutes les références renvoient à l'édition de La Comédie humaine dans la Pléiade », et en fin de volume, la récapitulation - comme l'affichage d'une bordure balzacienne - de 18 pages de notes répondant aux appels figurant dans le texte qui, pour plus de $90 \%$ réfèrent précisément à un écrit de Balzac ${ }^{99}$, le texte lui-même est dominé par le dire autre de Balzac : toujours maximalement marqué $\left(\mathrm{V}_{6}\right)$ en DD, ou MAE, au fil du Discours, et souvent comme émancipé par une disposition paginale détachant (sans guillemets) par une marge plus importante, de larges coulées ${ }^{100}$ de plus d'une page parfois, de « morceaux choisis ».

À ce composant de base de la bordure (« attaché » à l'objet du Discours) s’ajoutent - occupant une bien moindre étendue ${ }^{101}$ - plusieurs strates de discours autres, diversement associées au Discours. La première, propre à l'inscription du texte dans l'espace des "études littéraires », et qu'ignore conséquemment le rituel (2.4.b) des Discours à l'Académie Française, consiste en renvois, généralement brefs, de formes variées, $\mathrm{DI}, \mathrm{DD}, \mathrm{MAE}$, toujours précisément référencés $\left(\mathrm{V}_{3}\right)$ : renvois à quelque pairs choisis dans l'immense interdiscours des études balzaciennes (et partageant donc le même « objet »). La seconde fait place à quelques écrivains qui - hormis Proust, associé « obligé » par les échos qui se font entre ces deux peintres de la vie mondaine - font tous partie, Stendhal, Barbey, Chateaubriand, J. Gracq..., de la société choisie de l'auteur ou de son panthéon personnel ${ }^{102}$.

La dernière strate de la bordure porte, elle, au plus haut degré, dans ses « associations » à des discours autres, la dimension de choix apparue avec la précédente; liberté, fantaisie, caprice, légèreté... caractérisent autant le choix des autres - disparates, imprévisibles, insolites... - que la forme cavalière de leur référenciation dans l'interdiscours $\left(\mathrm{V}_{3}\right)$ : au fil du Discours surgissent ainsi, au gré de l'énonciateur, sans autre " raison » que celle de son bon plaisir, de ses goûts et de ses associations d'idées : Lautréamont, Mallarmé, Artaud, Chardonne, etc., sans alourdir de références précises et d'appels de notes... ces émergences, les divers degrés de désinvolture dans le marquage faisant plus ou moins appel à la connivence :

99 Notes que redouble l'ensemble (10 pages) strictement balzacien des index des œuvres et des personnages évoqués dans l'ouvrage ; cependant qu'une seule double page d'« Éléments de bibliographie » suffit à insérer l'ouvrage dans un environnement interdiscursif restreint.

100 Dont la liaison avec les mots de Ph. Berthier offre le plus riche répertoire de $\mathrm{DD}$ « annoncés » ( $v s$ « construits », $c f$. chap. 8) et des formes de continuité syntactico-énonciative de MAE.

101 Une trentaine de formes au total, là où c'est par centaines que se comptent les omniprésents fragments de Balzac.

102 Comme en atteste, en tête d'ouvrage, la rubrique « Du même auteur », mentionnant les études que celui-ci leur a consacrées. 
- $\quad l$ et guillemets :

[...] la frivolité - « état violent », comme le dira Oscar Wilde (p. 117)

La province, dirait Lautréamont, c'est « tics, tics et tics » (p. 250)

- $\quad l$ sans guillemets :

Si, comme l'a affirmé Chardonne, l'amour c'est bien plus que l'amour, Balzac c'est bien plus que Balzac (p. 312)

- guillemets sans $\boldsymbol{l}$ :

[...] un jeune poète peut-être génial, « suicidé de la société » (et quel suicidé ne l'est pas ?) (p. 310)

- et surtout, en abondance, degré zéro de l'allusion :

Aboli bibelot d'inanité sonore, le parlage provincial, en roue libre, dévide machinalement ses pseudo-échanges. (p. 250)

Mais là encore, la pesanteur est souvent plus forte que la grâce (p. 246)

Il ne reste plus désormais qu'à [...] reconquérir les provinces mondaines perdues [...] le regard crânement fixé sur la ligne bleue des salons. (p. 263)

b) Au cœur du rituel de cooptation d'un nouveau membre à l'Académie Française, les deux discours qui se répondent- discours d'accueil célébrant l'impétrant, discours d'éloge, par celui-ci, de celui qu'il remplace - illustrent cette même configuration de RDA d'un centre " assigné » $\left(\mathrm{V}_{4}\right)$ à celui dont on parle, ici dans une tonalité $\left(\mathrm{V}_{5}\right)$ positive, qu'accompagne une frange vagabonde offerte aux autres librement associés $\left(\mathrm{V}_{4}\right)$.

$\mathrm{Au}$ fil de l'histoire de l'Académie, c'est un passionnant corpus de $\mathrm{RDA}^{103}$ qu'offre cet ensemble de discours hypernormés, précisément par la mise en œuvre de ce parquoi chacun se spécifie : le choix des formes $\left(\mathrm{V}_{6}\right)$ de la RDA « contractuellement » attachées $\left(\mathrm{V}_{4}\right)$ à l'objet et celui, sans restriction, des voix autres diverses appelées à se mêler (associées $V_{4}$ ) à son propos - porteurs des effets et stratégies les plus divers dans l'accompagnement qu'elles font - consonnant, complice, distancié, discordant - à la ligne dominante et obligée des panégyriques.

$103 C f$. le précieux travail de M. Kolopp (1997) sur lequel je m’appuie. 
Je n'en prendrai qu'un exemple ${ }^{104}$, lié au hasard des successions, qui confie à un poète surréaliste, Roger Caillois, la charge de faire l'éloge d'un historien de l'Antiquité, Jérôme Carcopino, dont rien - l'œuvre abondante et érudite, la carrière universitaire et administrative, marquée d'un épisode vichyssois - ne le rapproche. Caillois remplit son contrat par la place prépondérante qu'il fait à la RDA de son prédécesseur : mais - très significativement par rapport à d'autres discours $^{105}$ - c'est massivement par des formes $\left(V_{6}\right)$ non autonymisantes (DI, MAS) ${ }^{106}$, celles-ci étant, pratiquement, confinées dans la mention des titres d'œuvres, prêtant ainsi minimalement sa voix aux mots de l'autre ; et le bouquet composite des voix amies - Borgès, Pascal et Picasso, Saint-John-Perse, l'Ecclésiaste, etc., et Rimbaud, longuement, pour finir - que, convoquées en DD, MAE, il associe $\left(\mathrm{V}_{4}\right)$ au sens fort à sa parole, ouvre - peu accordé à celui dont l'éloge lui est échu - sur la liberté d'un espace personnel.

\section{Variable $\left(V_{3}\right)$ : degré de spécification des coordonnées réfé- rentielles du discours autre}

Par spécification des coordonnées référentielles du $\boldsymbol{a}$ représenté, ce qu'on vise ici est limitatif par rapport (a) à ce qui a été évoqué (chap. 5.3) comme contextualisation représentée et $(b)$ au degré de précision de la représentation de l'énoncé lui-même. Ainsi un énoncé du type :

Hier matin, à sa descente d'avion, l’actrice a échangé des propos aimables avec le metteur en scène.

où $\boldsymbol{l}, \boldsymbol{r}$ sont identifiés, temps et lieu de l'acte de parole spécifiés, sera considéré comme présentant « un degré élevé » de spécification des coordonnées de l'acte représenté, alors que la représentation du contexte (a) - nécessairement « incomplète » - peut être estimée " insuffisante » (Quelles sont les relations entre les deux personnes ? Quel est l'enjeu de cette rencontre ? etc., toutes choses susceptibles de donner du sens aux « propos aimables »), et minimale la représentation des énoncés eux-mêmes (b).

104 Janvier 1972. Éloge par R. Caillois de son prédécesseur J. Carcopino.

105 Celui de son accueil à la même séance par R. Huyghe, ou celui par lequel, chaleureux, admiratif et complice, il accueillera C. Levi-Strauss (juin 1974).

106 Passant de surcroit préférentiellement par des formes de DI « catégorisantes » (cf. chap. 6.4, ou réduites au « thème » du discours, chap. 7.3) au détriment des reformulations en « Vparole que $P$ ». 
Ce qui est visé ici, c'est le degré d'identification de la source $\boldsymbol{l}$ d'un acte $\boldsymbol{a}$, se situant entre les pôles où se situeraient, par exemple :

Les derniers mots de Goethe ont été : « Mehr Licht ».

de l'acte de parole spécifié d'un $\boldsymbol{l}$ identifié, et

Il y a des gens aujourd'hui qui soutiennent que la terre est plate.

d'actes de paroles non situés dans l'espace et le temps, émanant de sources $l$ indéfinies.

Le degré de spécification du discours autre représenté présente une pertinence aux plans des genres, des discours, comme facteur de caractérisation différentielle globale entre les Discours d'une part, et dans les Discours d'autre part, comme facteur de différenciation interne à chacun entre leurs discours autres.

\subsection{Pertinence différenciatrice entre genres, domaines ou sphères discursives}

Sur une échelle de spécification décroissante, on peut, par exemple, situer (en renvoyant largement à des cas déjà rencontrés) comme relevant d'un degré

(i) maximal:

- les genres de « tenant lieu » à statut juridique, ou impliqués dans des parcours fortement contraints : procès verbaux, compte rendus de séance officielle, minutes de procès, rapports de psychiatres ou d'assistantes sociales ;

- les genres des «Questions au gouvernement » ${ }^{107}$;

- les « références » complètes requises par le discours scientifique, comportant $\mathrm{N}$ propre et « adresse interdiscursive » précise ;

(ii) intermédiaire:

- la vulgarisation scientifique, pour laquelle contrairement au genre précédent, c'est le flou des indéfinis ou des Npropres sans « adresse interdiscursive » qui est de mise : les experts, la communauté scientifique, le professeur $X$;

107 Cf. ci-dessus chap. 5 ex. (25), p. 163. 
- le « genre » de l'exergue, ne spécifiant, normalement, que le nom propre de l'auteur ;

- les « citations d'auteur » dans les Dictionnaires de langue ${ }^{108}$, les recueils de citations sur des mots, des notions, les florilèges sur un thème ;

- le genre du sermon, analysé par Maingueneau (2010) ${ }^{109}$, privilégiant, pour le recours aux textes sacrés, la référence aux seuls $\mathrm{N}$ propres, significativement dépourvus de l'historicisation désacralisante d'indications spatio-temporelles ;

- les genres visant à « éduquer » les gens, les parents, les enfants, l'enseignant, l'élève, le mari, le patron, etc., et prônant ou dénonçant des façons de dire propres à ces entités génériques ;

(iii) minimal:

- dans le champ de la connaissance, les manuels préférant l'énoncé de vérités établies $(P$, on sait que « $P$ ») à la référence à des sources ( $l$ a montré, selon l...) ;

- les Dictionnaires de proverbes, c'est-à-dire recueillant les énoncés issus de l'immémoriale « sagesse populaire » ou " des nations », c'est-à-dire sans source aucune.

Ils rejoignent en cela les "Dictionnaires des idées reçues » recueillant le dire anonyme de la doxa (contrairement au plan de leur énonciation, en discours, où proverbes et stéréotypes se distinguent).

Remarque 5 : Le dire des proverbes et des idées reçues. Les idées reçues peuvent être énoncées comme telles, c'est-à-dire représentées (RDA), à distance, avec ironie ou adhésion, mais, le plus souvent elles sont incorporées au discours comme son tissu même. En revanche l'énonciation d'un proverbe, énoncé saillant reconnu comme tel, relève foncièrement de la RDA comme autre associé $\left(\mathrm{V}_{4}\right)$ au Discours ; cette énonciation passe par les modes autonymisants du DD ou de la MAE, sous des formes diverses : (1) à marquage zéro (DDL, allusion), (2) purement typographiques, (3) avec « explicitation » d'une source, comme annulée dans l'indéfinition du « on » (on dit, comme on dit, on a raison de dire [que]...) ou « court-circuitée » dans la réflexivité de le proverbe dit, comme dit le proverbe.

108 Le degré zéro de la spécification s’atteignant avec l'épuisement de la notion d'acte de parole, dans le « on dit » d'un fait de langue : « En français on dit : "traverser la rivière en nageant” là où l'anglais dit : "To swim across the river" ".

109 Cf. ci-dessus chap. 5.5.2.2, p. 185 ; le recours aux « grands associés » $\left(\mathrm{V}_{4}\right)$ - Mao, Lacan, Der Führer, ou « papa m’a dit »- empruntant volontiers ce même mode d'appel global à une autorité alors même qu'on en cite des mots. 
Parfois accompagnée de distance ironique par les énonciateurs, cette énonciation de proverbes est volontiers représentée (RDA dans une RDA) comme apanage de personnages (théâtre, roman) ridicules, et symptôme de leur « bêtise » (au moins apparente). Qu'on pense à ce qu'A. Roger, dans son Bréviaire de la bêtise ${ }^{110}$, appelle les « crises » ou les « bouffées » proverbiales de Sancho Pança, aux batteries de proverbes et de tautologies que Sganarelle tente d'opposer à la raisonneuse ironie de Don Juan, comme dans la tirade ${ }^{111}$ commençant par

Sachez, Monsieur, que tant va la cruche à l'eau, qu'enfin elle se brise; et comme dit fort bien cet auteur que je ne connais pas, l'homme est en ce monde ainsi que l'oiseau sur la branche [...].

ou encore Figaro raillant Bazile ${ }^{112}$ :

Ah ! voilà notre imbécile avec ses vieux proverbes ! hé bien ! pédant, que dit la sagesse des nations?

\subsection{Pertinence différenciatrice relevant de choix singuliers}

Les exemples sont multiples d'un degré de spécification relevant, non pas d'une prescription générique mais d'un choix énonciatif singulier ${ }^{113}$.

- Relevant d'un degré élevé de spécification, on peut évoquer l'expérimentation littéraire d'A. Frontier dans Portrait d'une dame, jouant du contraste entre des coordonnées - identité, jour, heure... - « anormalement » spécifiées et l'absence de contextualisation, situationnelle et dialogale, livrant les mots «sans filet »...

- À l'inverse le discours de la ville - entendu, lu sur les murs... - qu'orchestre Döblin dans Berliner Alexander-Platz est celui d'une multiplicité anonyme, à laquelle Butor donne la forme d'une houle roulant au pied des murs de la Place Saint-Marc à Venise.

- Se répondant, sous la plume d'un même auteur, Annie Ernaux, on a évoqué114 le discours autre représenté maximalement spécifié - identité de $\boldsymbol{l}$, lieu, jour de « Elle », sa mère, aux paroles « recensées » une par une et, à l'inverse, dans

110 Roger (2008: 73-80), dans un séduisant parcours posant le « c'est comme ça » et le « depuis toujours » comme « les deux piliers de la bêtise rustique du proverbe » se présentant comme le dépôt d'une expérience immémoriale ».

111 Molière, Don Juan, acte V scène 2, « sublime en son genre (la bêtise) », dit A. Roger (2008 : 78).

112 Beaumarchais, Le Mariage de Figaro, acte I scène 11.

113 Je privilegie ici les textes déjà rencontrés chap. 5.5.2.1, p. 184 notamment.

114 Cf. ci-dessus 2.1, p. 601. 
Les Années, le mouvement d'un discours qui se produit, sans actes d'énonciation spécifiés - source, temps, lieux indéfinis d'un " on disait »...

- Se faisant vis-à-vis, dans un même contexte (discussion à l'Assemblée Nationale au sujet de l'abolition de la peine de mort), ce sont aussi deux énonciateurs dont les positions idéologiques opposées s'appuient, on l'a $\mathrm{vu}^{115}$, d'un côté sur le discours de l'opinion, « des gens », pour, de l'autre, faire appel aux paroles, fortement spécifiées, de « grands hommes ».

\subsection{Pertinence différenciatrice dans un discours, texte.}

Les effets internes à un Discours qu'inscrit une différence dans le degré de spécification des autres discours qu'il accueille, se situent sur plusieurs plans.

\subsubsection{Structuration d'un récit fictionnel}

Deux plans de RDA à degré de spécification opposé - celui de personnages déterminés s'exprimant à des moments particuliers de l'histoire $v s$ celui de la voix au contours imprécis de l' « Histoire » ou « des gens » - peuvent s'articuler, diversement, dans la voix narrative d'une fable, d'un conte, sur un mode globalement structurant.

Différant en cela de la plupart des fables de La Fontaine où nous entrons de plain-pied dans le récit - directement énoncé par le fabuliste - des aventures et propos de « Maître Corbeau » ou du «Souriceau tout jeune... », certaines s'ouvrent par la référence à une autre voix qui précède et d'où procède celle du fabuliste. C'est alors l'ensemble du récit qui se trouve inséré dans une structure de RDA, enchâssé dans on dit que ou j'ai lu dans... que $e^{116}$ :

Les Levantins en leur légende / Disent qu'un certain Rat... (VII-3) ; On conte qu'un serpent... (V-16) ; J'ai lu chez un conteur de fables... (III-18) ; J'ai lu dans quelque endroit... (III-1) ;

modalisé en assertion seconde par à ce qu'on dit... :

[...] la poule, à ce que dit la fable / Pondait tous les jours un œuf d’or... (V-13) ;

115 Cf. chap. 5.5.4.2, p. 191, 11.3 (48), p. 462 et Micheli (2006, 2007).

116 Cadrage initial qui peut, comme dans III-1 (« J'ai lu en quelque endroit »), être redoublé, deux vers plus loin, par un «si j'ai bonne mémoire ». 
ou accompagné d'une incise ${ }^{117}$ :

Un envoyé du Grand Seigneur / Préférait, dit l'histoire... (I-12) ; Les animaux [... ] s’assemblèrent, dit-on (VI-6) ;

le récit prenant place dans une vaste mémoire dont le fabuliste se fait le transmetteur.

Ce peut être aussi, non en cadrage initial de toute la fable mais en touche légère, que surgit ponctuellement dans le récit, en incidente, le rappel du vaste « avant et ailleurs » discursif dont la fable s'est détachée ; ainsi :

Certain Renard gascon, d'autres disent normand, / Mourant... (III-5)

ou seulement au terme (ici fatal) de l'errance " grimpante » des Deux Chèvres entêtées de prestige nobiliaire

[ayant] la gloire / De compter dans leur race, à ce que dit l'histoire,/ L'une certaine chèvre... (XII-4).

Plus insolite ${ }^{118}$ est le jeu, à deux RDA, inégalement spécifiées que dégage J.-M. Adam (2006b : 41) dans le genre du conte : l'apparition chez Perrault, « aux frontières du conte ", - lorsque s'achèvent les aventures de Riquet à la Houppe ou du Petit Poucet, avec toutes les paroles, les dialogues dont elles s'accompagnent de la voix de « quelques-uns » ou de « bien des gens » qui « disent », " assurent », etc., bref commentent l'histoire qui précède. Ainsi se succèdent immédiatement deux plans de RDA, celui de ${ }^{119}$ :

La Princesse n'eust pas plus tost prononcé ces paroles que Riquet à la houppe parut à ses yeux l'homme du monde le plus beau [...] qu'elle eust jamais vu

et celui de :

Quelques uns assurent que ce ne fut point les charmes de la Fée qui opérèrent, mais que l'amour seul fit cette métamorphose. Ils disent que [...], que [...] et que [...] : [...].

117 Qui, $c f$. chap. 9.3.2.2, p. 351, neutralise l'opposition $\boldsymbol{l}$ dit/selon $\boldsymbol{l}$.

118 C'est-à-dire ne relevant pas de la classique structure d'emboîtement de récits dans laquelle $\mathbf{L}$ inscrit, dans la dépendance d'une autre voix $\boldsymbol{l}$, le récit - faisant figurer d'autres autres $\left(\boldsymbol{l}_{1}, \boldsymbol{l}_{2} \ldots\right)$ qu'il tient de la première $(\boldsymbol{l})$.

119 Cités d'après Adam (2006b : 41). 
En passant du récit à RDA bien spécifiée ( $\boldsymbol{l}$ identifié, actes situés) des personnages, à la représentation imprécise ( $\boldsymbol{l}$ indéfini, actes non situés) de commentaires au sujet du récit, la voix narrative n'enchâsse pas son récit dans un cadre plus vaste qui, homogènement, l'englobe, mais - « surprenant changement de cadrage énonciatif » dit Adam - se déporte dans un ailleurs discursif, problématique, hétérogène au fonctionnement du conte.

\subsubsection{Classer les autres d'un Discours par leur degré de spécification}

Le classement - volontiers hiérarchisant, voire axiologique - des discours autres par leur degré de spécification s'observe dans des genres, des champs divers et aussi dans des textes singuliers.

On a évoqué $\mathrm{e}^{120}$ - comme propre à un ouvrage de " psychologie du bonheur » et au genre dont il est un représentant, et comme essentielle dans l'image qu'il veut donner de lui -, la forte structuration en trois « cercles » d'autres, définis par le type et le degré de leur spécification : celui, minimal, de la voix anonymisée de patients, celui des noms illustres, incarnation de pensée profonde et d'humanité, sans référence précise, celui, enfin, des experts, garants de la scientificité où s'ancre le discours, cités avec toutes les normes requises.

Dans le champ des Sciences Humaines l'écart est clairement saillant entre la représentation de la « voix du terrain », orale, des « informateurs » - très majoritairement indéfinis : « on, un habitant, un ancien, un résident, les gens, la population » - et celle « de la science » écrite, des publications émanant des autorités et des pairs, maximalement référencées ${ }^{121}$.

A. Bolón (1996 : 197-207) fait apparaître comment, dans un long article du journal Le Monde ${ }^{122}$, intitulé « Les petits frères des beurs », s'opposent deux types de représentation de discours émanant, respectivement :

- d'un ensemble d'énonciateurs, chacun désigné par son Nom propre, souvent assorti d'indications sur son statut social et son expérience, tels, par exemple :

[...] le sociologue Adil Jazouli qui vient de visiter une quinzaine de banlieues " chaudes» de Lyon, Marseille et Paris [...] ; Christian Delorme, que l'on surnommait « le curé des Minguettes » [...] ; Andrée Chazalettes, déléguée du Fonds d'Action Sociale dans la région Rhône-Alpes [...] ;

120 Chap. 5.3.2, p. 161 et chap. 12.2.1, p. 479.

121 Je me fonde, notamment, sur l'étude précise par M. Ossard (1995), d'un numéro de la revue d'ethnologie Terrain, consacrant sous le titre « Boire » (n¹3, octobre 1989) 18 articles à la diversité - en France et ailleurs - des pratiques sociales de boisson.

122 Le Monde, 11-10-1990. 
- $\quad$ et de la collectivité des " petits frères des beurs ", apparaissant comme pluralité anonyme, principalement en ils (moins souvent en certains, une fois en « ces beurs ») :

a) [...] ils sont passés à la télé, et d'ailleurs s'en vantent : ils ont fait reculer les forces de l'ordre...

b) [...] soucieux de leur « look », ces beurs n'ont que mépris pour les « loques » que sont à leurs yeux les drogués...

c) Ils se ne reconnaissent pas en Harlem Désir, « c'est un Bounty, noir dehors, blanc dedans » lancent ironiquement certains.

Remarque 6 : Degré de spécification et formes de RDA $\left(\mathrm{V}_{6}\right)$. On notera que, dans ce texte, la différence dans la spécification des énonciateurs se double d'une opposition dans les formes de RDA mises en œuvre : DD assortis, en incise, de verbes comme disent, soulignent, remarquent du côté des observateurs ; diversité de formes de l'autre côté - des « observés »-, ayant pour résultat commun de «ne pas laisser la parole aux énonciateurs » : en la reformulant largement (a-b-c); en y pointant des «manières de dire » (b) ; et plus remarquablement en faisant intervenir (en c) un DD qui, préalablement reformulé par $\mathbf{L}$, est, largement vidé de son statut d'événement de parole porteur de sens, réduit à la monstration d'une manière de dire ${ }^{123}$.

On retrouve cette corrélation entre spécification de la source $\left(\mathrm{V}_{3}\right)$ et précision de représentation des énoncés $\left(\mathrm{V}_{6}\right)$ dans Les Rêveries du promeneur solitaire, où s'opposent deux versants de RDA : le premier est celui du souvenir évoqué par Rousseau de ses propres propos - représentés précisément dans leur singularité, de façon émue ou pittoresque (au DD, DI) - et parmi eux, dans la dernière promenade, écrite " Aujourd'hui jour de Pâques fleuries » anniversaire des cinquante ans de sa rencontre avec Madame de Warens, dernière parole que le texte évoque, qui devait ouvrir sur « le bonheur de sa vie » : « j'engageai maman à vivre à la campagne »; le second, maximalement indifférencié, du discours « du complot» ravalé à l'indistinction d'énonciateurs pluriels anonymes - mes persécuteurs, mes ennemis, tous les philosophes, l'opinion... - incessamment représentés en ils et $e u x$, et de propos réduits (touchant à la limite de la RDA qui requiert la représentation d'un contenu) à leur catégorisation comme « offenses, outrages, arguties, sophismes, insensés jugements des hommes... »

123 «On produit une absence d'énonciation en montrant leurs mots » (p. 206), faisant de la parole de ces énonciateurs une « absence d'événement » (p. 202), analyse A. Bolón (1996) qui rejoint ce que Voloshinov (1929/1977 : 185 sq.) décrit comme « discours direct vidé de sa substance »; voir ci-dessus chap. 9 notes 63 et 64, p. 357. 


\subsubsection{Effets de perspective et contrastes dans le roman}

Le jeu de l'alternance entre discours indéfini - bavardage, caquetage, brouhaha, bruit, rumeur... - d'une collectivité, de « tous », de la foule, de l'opinion, du « on ${ }^{124}$, et la parole singulière prêtée à un personnage, est un ressort de l'écriture romanesque.

Victor Hugo l'orchestre dans Les Misérables ${ }^{125}$ lorsque, à l'errance à travers Digne de Jean Valjean, rejeté de partout dans sa quête d'un repas et d'un refuge pour la nuit (chap. 1), répondent, successivement, la rumeur de la ville dont Madame Malgloire se fait l'écho auprès de Monseigneur Myriel - morceau de bravoure de non-spécification des discours représentés :

[elle] avait entendu dire des choses en divers lieux. On parlait d'un rôdeur de mauvaise mine ; qu'un vagabond suspect serait arrivé, qu'il devait être quelque part dans la ville, et qu'il se pourrait qu'il y eût de méchantes rencontres [...]. Que c'était donc aux gens sages à faire la police eux-mêmes et que [...] Il paraîtrait qu'un bohémien, un va-nu-pieds, une espèce de mendiant dangereux serait en ce moment dans la ville. Il s'était présenté pour loger chez Jacquin Labarre qui n'avait pas voulu le recevoir. On l'avait vu arriver [...] Tout le monde le dit.

et l'échange de paroles, aussi spécifiées qu'il est possible, de l'évêque et de Jean Valjean :

En ce moment, on frappa à la porte un coup assez violent.

- Entrez, dit l'évêque. (fin du chapitre 2)

Un homme entra [...] et dit d'une voix haute :

- Voici. Je m’appelle Jean Valjean. Je suis un galérien [...]

-Monsieur, asseyez-vous et chauffez-vous. [...] Je suis, dit l'évêque, un prêtre qui demeure ici.

Cette opposition se prête à de multiples effets de rencontre - en un lieu, un temps... - où, par contraste, les paroles ou pensées d'un héros se détachent, individualisées, sur fond de propos anonymes. Flaubert, maître ès circulation du discours commun de la « bêtise », en use régulièrement (même si, chez lui, la parole de ses héros est largement ventriloquée par cette même grande voix...) : ainsi, dans le long récit de la soirée chez les Dambreuse ${ }^{126}$, est-ce par deux fois que, d'abord dans le vaste salon où se presse la foule des invités, puis dans l'espace plus restreint du boudoir de la maîtresse de maison, les propos rapportés sur-

$124 C f$. par exemple les effets de parole « de groupe » chez Flaubert ou C. Simon (chap. 6 (46), p. 222, chap. 7 (49), p. 244).

125 Partie I, Livre II, chap. 1, 2, 3.

126 L'Éducation Sentimentale, (IIème partie, chap. 2), p. 199-207 (éd. Société Les Belles Lettres) 
viennent, dans leur insignifiance anonyme, comme simple émanation des lieux, dont la description - silhouettes et décoration - passe par toutes les ressources de la langue en matière d'indéfini pluriel ${ }^{127}$; ainsi, placé non loin de quelques quadrilles,

Frédéric entendait des phrases comme celles-ci :

- «Avez-vous été à la dernière fête de charité de l'hôtel Lambert, Mademoiselle ? »

- «Non, Monsieur!»

— « Il va faire, tout à l'heure, une chaleur !»

- «Oh ! c'est vrai, étouffante » [...]

Et, derrière lui, [...] d'autres causaient chemins de fer, libre-échange ; un sportman contait une histoire de chasse ; un légitimiste et un orléaniste [...] discutaient.

cependant que, dans le boudoir :

le murmure des voix féminines, augmentant, faisait comme un caquetage d'oiseaux.

Il était question des ambassadeurs tunisiens et de leurs costumes. Une dame avait assisté à la dernière réception de l'Académie ; une autre parla du Don Juan de Molière, représenté nouvellement aux Français.

Paroles indifférenciées, interchangeables... c'est sur ce fond que survient, et tranche, un vrai dialogue, suivi, entre Frédéric et le banquier ${ }^{128}$ - dont le dernier échange fait soudainement, « comme dans un éclair » apercevoir au jeune homme " une immense fortune qui allait venir».

La scène des deux amants de Belle du Seigneur soumis, dans le hall de l'hôtel où ils se tiennent, au fond sonore du discours des « tricoteuses ${ }^{129}$, fait, sur un mode tragi-comique, contraster, par leur simultanéité, ces deux images, définie et indéfinie, du dire : la parole personnelle, fragile jusqu'au silence, des héros, enfermés dans leur " souricière d'amour », dont les tentatives de dialogue échouent dans le repli muet de leurs pensées :

$127 \mathrm{Cf}$. « La foule des hommes », " quelques dandies », " deux ou trois médecins illustres », " de petits jeunes gens à la barbe naissante ", " des femmes assises en rond tout autour d'un pouf », " une valetaille à longs galons d'or », " trois autres salons qui regorgeaient d'objets d'art », sans oublier l'admirable « entrée » dans le boudoir de Madame Dambreuse : « Des femmes le remplissaient les unes près des autres, sur des sièges sans dossier ».

128 Où, au DD, DI, DIL, ils « affirment, répondent, objectent, reprennent... ».

129 Belle du Seigneur, chap. 87, édition Folio, p. 852-860; cf. ci-dessus chap. 5.5.2.1, p. 183. 
[...] dit-elle [...] elle lui demanda [...], pensa-t-il [...] il acquiesça [...]. À voix basse, elle lui demanda de lui donner la main, lui dit qu'il était tout pour elle, tout. C'est vrai, d'ailleurs, pensa-t-il, et elle tout pour moi, et cela nous fait une belle jambe.

et le flot ininterrompu de « fragments disparates, puissantes litanies » qui s'écoule, implacable, de « bouches [... qui] remuaient sans répit », et dont les deux amants « se tenant par la main » sont réduits à « écouter » ce qui leur parvient des « duos emmêlés »...

Flaubert va plus loin encore - lors des «Comices agricoles » - avec la rencontre cacophonique qu'il met en scène, entre les fadaises que Rodolphe murmure aux oreilles d'Emma et la sonore annonce anonyme relative aux cochons et fumiers ${ }^{130}$.

L'alternance entre paroles énoncées singulièrement et discours porté par réparti sur - une pluralité de sources anonymes peut prendre une autre résonance lorsque, comme dans Nous étions des êtres vivants de N. Kuperman ${ }^{131}$, la représentation en forme de « chœur » d'une énonciation collective en « nous » ou en « on » de l'ensemble du personnel de l'entreprise, fort d'une expérience commune et d'une appréhension lucide de la « restructuration » qui les menace de licenciement, rassemble et dépasse la dispersion des propos tenus par chacun, aux prises isolément avec sa façon - enjeu et stratégie individuelle - de réagir à la crise qu'il affronte ${ }^{132}$.

\subsubsection{Un texte structuré par l'opposition spécifié vs non spécifié}

L'essai de Richard Millet « Désenchantement de la littérature » "133 est une inquiète, sinon désespérée, déclaration d'amour à la littérature - comme pratique singulière du langage, rapport « fervent » à la langue, « écart réfutant le langage mortifère de la communication » (p. 17) -, doublée de la plus véhémente diatribe contre le règne d'un fonctionnement communicationnel aux couleurs de l'anonymat et de la réduction de la langue à un statut « instrumental, véhiculaire » (p. 60).

Cet affrontement manichéen trouve sa forme dans l'opposition, traversant le texte de part en part entre les valeurs extrêmes - intensément et explicitement axiologisées ${ }^{134}$ - de la Spécification $\left(V_{3}\right)$, les deux discours autres, respectivement

130 Cf. chap. 5, exemple (72), p. 182.

131 Gallimard NRF, Paris, 2010.

132 B. Bloch (2012 : 216-221) analyse précisément le fonctionnement de ce tissu narratif et énonciatif original.

133 Gallimard, Paris (2007).

134 Par un auteur observant, en avant-propos « [son] exécration de l'espèce humaine et [son] amour pour l'individu» (p. 12). 
révérés et honnis, de l'écriture littéraire et de la communication, occupant les deux pôles de la singularité et de l'indéfinition ${ }^{135}$.

D’un côté, celui des « ciels étoilés », de la " voie lactée » où brillent les " figures » emblématiques des "vrais écrivains », faisant entendre leurs voix singulières comme autant de modes individuels de "servir la langue ", invocation de noms propres divers ${ }^{136}$, appelés à chaque page ${ }^{137}$, isolément, par petits groupes, en longues énumérations incantatoires et récitation de leurs mots. $\mathrm{Si}$ les $\boldsymbol{l}$ sont individualisés, l'évocation - matérielle - de leur parole passe en effet quasi exclusivement par les modes autonymisants ${ }^{138}$ : amples coulées de DD de Hofmannsthal, Nietzche, Chateaubriand, Artaud ou Rilke, réénoncées, revivifiées par l'auteur et offertes au lecteur en partage régénérant ; brefs éclairs en MAE, traversant fréquemment l'écriture de l'auteur, des comme dit..., ce qu'appelle..., ou pour parler avec... :

[...] un esclavage volontaire, au sens de La Boétie, et qui me rendrait esclave de «l'oubli de l'être », pour parler encore avec Heidegger. (p. 27)

[...] le monde (comme dirait Wittgenstein) est une grammaire [...] (p. 27).

Ailleurs, du côté de l'autre discours, où dans un "enténèbrement du monde " (p. 61), « devant l'obscurité qui vient », « entrés dans un étrange hiver : celui de la langue », «le silence guette nos bouches » (p. 15), n’apparaissent ni énonciateurs individuels ni énoncés singuliers.

Aux chapelets de noms propres illustrant le premier espace succède le règne de l'anonymat, de l'interchangeabilité où la référence à des discours passe par des noms communs et indéfinis pluriels :

les communiquants (idt, p. 58) ; les chiens de garde (p. 18, 44); mes ennemis (p. 28), tous ceux qui ont abdiqué (p. 53) ; les zélateurs (p. 66) ; les vertueux (p. 25) ; les barbares des banlieues des grandes villes (p. 49); etc.

et le on, notamment dans cet énoncé où il permet à l'auteur de ne pas énoncer directement le mot - aussi détestable que la chose - de doxa :

135 C'est de cela qu'il est question ici, non de rendre compte de l'ensemble des thèses soutenues dans cet ouvrage, passionnément « réactionnaire », au sens propre du terme, et « anti-démocratique ».

136 Vivants et morts ; poètes, romanciers, philosophes ; de France et d'ailleurs que seule rassemble la ferveur de l'auteur : Céline et Montaigne, Peter Handke et Baudrillard, Valéry et Melville, W. Blake et Saint-Simon, etc.

137 Près de 200 occurrences de noms d'auteurs ou d'œuvres.

138 Rarissimes DI et MAS sans îlots textuels. 
[...] le vieux processus de récupération, de production d'anticorps, de recyclage et de falsification du réel qu'on appelle la doxa (p. 29).

L'indifférenciation des discours autres va, au-delà de son attribution à des sources humaines " indéfinies ", vers le traitement a- ou infra-énonciatif, si l'on veut, de ce discours saisi - aussi loin qu'il est possible d'une représentation de propos chargés de sens et portés par des sujets - comme phénomène, processus, courant :

le totalitarisme mou (p. 19) ; le langage démocratique (p. 28) ; le politiquement correct (p. 34) ; l'époque (p. 33) ; le dogme (p. 25) ; un ensemble d'oukases (p. 25) ; le diktat (p. 62) ; la pornographie sociale ayant décrêté que (p. 28); etc.

à travers les « supports », canaux, conduits par où il se propage :

la publicité ; le journalisme (discours, menace journalistique) ; la communication ; le mensonge médiatique qui fictionnalise le monde (p. 26) ; la visibilité ; les nouveaux appareils idéologiques (p. 25).

Ainsi cette imprécation continue contre une discursivité haïe parvient-elle, par le degré zéro de spécification qu'elle met en œuvre pour les discours évoqués - tant au plan des énonciateurs que des énoncés - non seulement à la séparer radicalement de l'énonciation souverainement singulière de la littérature, mais au-delà à mettre en doute l'appartenance de ces manifestations langagières au domaine des faits, actes, événements de parole (représentables comme tels).

\section{Note sur les variables non traitées}

Constamment croisées en parcourant $\mathrm{V}_{1}, \mathrm{~V}_{2}, \mathrm{~V}_{3}$, les trois variables :

$\mathrm{V}_{4}$ - du mode d'Émergence du discours autre comme attaché à l'objet $v s$ associé au cheminement du Discours,

$\mathrm{V}_{5}$ - du Rapport établi entre le Discours et le discours autre convoqué, $\mathrm{V}_{6}$ - des Formes de RDA par lesquelles le Discours s'articule à son autre, ne seront pas, on l'a dit, systématiquement parcourues.

Ce choix repose sur plusieurs points : l'espoir que les rencontres faites précédemment avec ces trois paramètres cruciaux du travail de bords en donnent un aperçu ; l'observation que le jeu permis par ces variables, notamment celui des tonalités et des formes que déploient $V_{5}$ et $V_{6}$, relèvent d'une logique plus complexe que celle suivie pour $\mathrm{V}_{1}, \mathrm{~V}_{2}, \mathrm{~V}_{3}$ et que l'exploration de son fonctionnement, non réductible à celui d'une variation par degrés, entre deux pôles, sur un axe, 
requiert de dégager d'autres itinéraires, dont le souci de contenir un texte déjà long dans des limites raisonnables ne permet pas d'emprunter...

\subsection{Variable $\left(\mathrm{V}_{4}\right)$ : émergence comme attaché/associé}

Plus aisément schématisable que pour les deux suivantes, le jeu de la variable $V_{4}$ (attaché/associé) présente déjà par rapport aux trois premières la complexité de ses deux régimes d'émergence - ou d'entrée - du discours autre dans le Discours. Chacun de ces deux versants fait apparaître une riche diversité de problématiques génériques, théoriques, esthétiques, subjectives liées à la $\mathrm{RDA}^{139}$.

Sur le versant du discours autre attaché à l'objet - outre le questionnement éclairant quant à ce qui, pour un Discours, peut être défini comme " son objet » (au sens non pas de sa « visée » mais de « ce dont il parle ») - apparaît, en fonction des types d'objet, selon qu'ils sont plus ou moins constitués de discours ou envisagés du point de vue de leur facette discursive, une riche variété de "réponses » des Discours à l'altérité du discours de leur objet : tels, notamment (outre le cas particulier des discours tenant lieu) critique littéraire, portraits, reportages, écrits de sciences humaines, récits (de faits réels ou fictifs), genres divers du dépaysement (romans exotiques, récits de voyage, guides touristiques)...

Sur l'autre versant apparaît comme fortement caractérisante pour les Discours la variété extrême de la place qu'ils font aux diverses figures de l'association :

- aux discours autres que l'on peut considérer comme "intrinsèques à l'énonciation » : discours propres aux je, tu, ici, maintenant, susceptibles d'émerger comme « autres » relativement au Discours en train de se faire ;

- à l'environnement discursif commun, aux clichés, proverbes...

- à des appuis dominants, à des compagnons familiers, à des ennemis insistants...

- à l'imprévu, au jeu de la mémoire et des associations d'idées.

139 De ces deux régimes d'émergence on peut noter que c'est très majoritairement du second que relèvent les exemples généralement proposés de «fonction » de la RDA dans le Discours ( $c f$. chap. 12 note 31, p. 478), comme si la fonction - massive - de la RDA de représenter le discours dont - ou de ce dont - on parle allait tellement de soi qu'il n'était pas nécessaire d'en parler... 


\subsection{Palette des Rapports $D / d\left(V_{5}\right)$ et « orchestre » des Formes $\left(V_{6}\right)$}

(i) Parmi les diverses variables, c'est sans doute celle $\left(\mathrm{V}_{5}\right)$ du rapport établi entre le Discours et les ailleurs qu'il accueille ou convoque dont les valeurs affleurent le plus immédiatement (bien plus que celles des variables $V_{2}$ ou $V_{3}$ de la Spécification et de la Dispersion) à la conscience du récepteur. Si s'impose évidemment la bipartition positif/négatif entre accord et désaccord - multiplement déclinée dans les analyses de textes en convergence/divergence, consensus/conflit, sympathie/antipathie, agonal/irénique, etc. ${ }^{140}$ - la palette des rapports $\mathrm{D} / \mathrm{d}$ mis en place est aussi étendue, diversifiée en nuances aussi innombrables que le sont les rapports entre êtres humains...

On rappellera seulement ici une facette propre à la RDA, que le Discours peut présenter dans son appel à un autre discours, jouant, en deçà des relations axiologiques à tel ou tel propos : celle, aisément empreinte de "sacralité ", d'une reconnaissance de dette ${ }^{141}$ - dette inhérente au langage même comme mémoire à transmettre $^{142}$, comme un leg ou un héritage - volontiers doublée d'une sorte de piété du re-dire comme mode de faire revivre la parole des morts. Dans un ouvrage consacré à la citation ${ }^{143}$, cette dimension de la RDA est soulignée comme relevant de la construction

[...] d'une collectivité faite de vivants et de morts [...] assurant à la fois l'indispensable séparation et une vivifiante circulation entre les vivants et les morts [...] un mouvement d'échange entre présents et absents, entre vivants et morts (p. 28).

Centrale est pour certains auteurs ${ }^{144}$, tel P. Quignard, - manifestée par « le geste citationnel [...] compulsif » que lui reconnaît F. Colin (2019: 108) - la tâche qu'il s'assigne, notamment pour les « auteurs délaissés » de l'Antiquité, de « faire revivre leur voix », " ramener ces oubliés à la vie », et qu'au-delà il reconnaît comme celle de la littérature :

$140 C f$., par exemple, les structurations d'un discours par sa RDA en termes de « dialogisme interdiscursif à visée convergente » vs « divergente » notées par M. Sandré (2011), " polyphonie consensuelle » vs « conflictuelle » analysées par Chetouani (2004).

141 Évoquée rapidement ci-dessus chap. 14.2.2.2.(i), p. 574.

142 Cf. M. Blanchot dans L'Entretien infini : «Qui s'intéresserait à une parole nouvelle, non transmise ? Ce qu'il importe, ce n'est pas de dire, c'est de redire, et dans cette redite, de dire chaque fois encore une première fois. "

143 Chambat-Houillon et al. (2004).

144 Mais aussi dans la vie privée, où l'emprunt ou le rappel des mots de « voix chères qui se sont tues » peut caractériser certains énonciateurs. 
Telle est la littérature. Chaque œuvre véritable repense tout ce qui a parlé, réanime tout ce qui s'est essoufflé, étouffé [...] éteint ${ }^{145}$.

(ii) $\left(\mathrm{V}_{6}\right)$ le jeu des formes

La question de la forme sous laquelle se réalise la RDA dans chaque occurrence ou, à l'échelle d'un Discours, dans l'ensemble des occurrences qui forment sa « bordure », entre évidemment de façon cruciale dans le « façonnage » de celle-ci. Le jeu des formes de RDA est une pièce essentielle du « travail de bords » : c'est à travers lui que se réalise le statut d'interface de la bordure, que se fait son «tissu», chaque occurrence de RDA est un fait de contact, rencontre, partage entre deux voix qui s'articulent - se mêlent, se superposent, se séparent, se confondent... selon les formes que propose la langue.

Si la zone de RDA d'un Discours peut être conçue comme la «bordure » dont il s'entoure, témoignant de son mode propre « d'être avec » l'altérité - celle des autres discours et, à travers eux, celle de l'extériorité du langage --, c'est en chaque occurrence de RDA que, selon la forme par laquelle à la voix effectivement parlante vient s'agréger, dans la linéarité, une voix autre, prend corps localement l'interface et se dessine un bord.

Deux remarques sur le jeu des formes de RDA dans le travail de bord.

Aucun des cinq modes distingués chapitre 9, abstraitement définis, chacun, par une Formule de traits différentiels et la zone de formes - à inventorier - par lesquelles il peut se linéariser, ne peut être assigné à un usage en discours, un effet de sens...

Ainsi la division énonciative propre au Bivocal-DIL - atteignant l'unité même du dire - si elle convient, et admirablement, à la représentation du discours intérieur, ne saurait certainement pas s'y réduire : la forme de langue, abstraite, de la bivocalité se situe en deçà des emplois auxquels elle se prête en discours. Et des rapports à l'autre discours aussi divers, par exemple, que celui d'un mimétisme ironiquement distancié à l'oral, ou du ton de neutralité sur lequel il est, dans les certificats d'internement psychiatrique du $19^{e}$ siècle, rendu compte de discours délirants, trouvent, dans la division énonciative, la forme de RDA qui leur convient.

On en dirait autant des autres modes, notamment du DD dont il faut se garder de rabattre le trait formel d'autonymie sur un des usages - la restitution fidèle auquel il se prête.

Seconde remarque : la moindre différence entre les diverses formes relevant de la « zone » d'un mode - dans son degré de marquage mais, au-delà dans toute

145 P. Quignard, Dernier Royaume, t. IX, Grasset, Paris, (2014 : 182), cité in Colin (2019 :109). 
variation - syntaxique, typographique, intonative... -, est susceptible d'être mise à l'œuvre comme pertinente dans le cadre d'un discours pour un travail différentiel des bords qui lui est propre (en fonction, par exemple, des divers $\boldsymbol{l}$, des rapports établis avec eux, de la nature orale, écrite, intérieure... du discours représenté, etc.). Ceci appelant à répertorier finement, pour chaque mode, le « clavier » qu'il propose, et dont jouent les Discours de façon spécifique - au-delà du jeu différentiel entre les modes que présentent, évidemment, les Discours, dans leur bordure - .

\section{Variable $\left(V_{7}\right)$ : bordure en mouvement}

La bordure de RDA que se donne un Discours, un texte, n'est pas nécessairement stable d'un bout à l'autre, ni (1) dans l'espace (temps) où il se déroule, ni (2) dans le temps de l'histoire d'un texte.

\subsection{Variation de la RDA dans le déroulement d'une unité textuelle}

Autant que la variété de langue mise en œuvre ou le rythme des phrases, etc., le jeu de la RDA peut être partie prenante du mouvement qui anime le texte (écrit ou oral), en termes de localisation ou d'évolution dans son avancée.

\subsubsection{Topographie textuelle de la RDA}

C'est tout au long de son déroulement que le fil du discours peut s'accompagner - « dialogiquement »- des autres qu'il accueille... mais il est aussi des lieux prédisposés à la rencontre - interface - entre le dedans et le dehors.

\section{(i) Dans l'espace textuel}

Apparaissent, situés génériquement « en bordure » de texte, principalement en ouverture :

- des lieux assignés à la RDA : celui qu'occupe le discours autre, très librement « associé » $\left(\mathrm{V}_{4}\right)$, de l'exergue-épigraphe, et celui, isolé en préambule, du rappel des « considérants ${ }^{146}$ sur lesquels se fonde la légitimité de l'acte de parole à valeur de loi qui les suit ;

146 Cf. 1.2 Remarque 4, p. 592. 
- des lieux prédisposés à l'accueil de RDA des titres, chapeaux et débuts d'articles de presse (surtout au DD), à fonction « d'accroche » comme aussi de l'amorce des «Questions au gouvernement » ${ }^{\mathbf{1 4 7}}$;

- des lieux « sensibles » du roman, catalysant « l'effet RDA » de DD, survenant de nulle part, de l'incipit et, plus souvent, ouvrant sur l'inconnu, des fins ${ }^{148}$.

\section{(ii) Dans l'espace matériel}

$\mathrm{Au}$ plan de la réalisation matérielle typographique d'un texte, ce qui retient l'attention, c'est l'affinité de la RDA avec les zones qui, au sens concret du terme, bordent le corps du texte, l'entourent, des «marges » de ses notes « de page ${ }^{149}$, de fin de chapitre ou de livre, et des « marches » territoriales de la bibliographie et des index ${ }^{150}$.

Comme une figuration matérielle du rôle de bordure-interface de la RDA assurant au Discours un contour propre dans l'extériorité discursive par l'identification de ses autres, c'est toute cette périphérie ${ }^{151} \mathrm{du}$ corps du texte « à cheval » sur son dedans et son dehors qui se prête spécifiquement (les notes) ou se consacre (bibliographie, index) au recensement et à la référenciation des extérieurs discursifs que se reconnaît le Discours.

Touchant l'affinité entre notes de bas (ou en marge) de page et RDA je renvoie à la précieuse étude de J. Lefebvre (2007) faisant notamment apparaître la variété des « répartitions » de RDA que présentent les textes, entre « corps » et « bordure » de notes - dans l'un ou l'autre de ces deux espaces paginaux ou à cheval sur les deux - comme une composante significative de leur écriture.

Quant à l'ensemble clôturant un texte - un livre - d'une bibliographie de ses " auteurs cités », ou, plus largement, " consultés », et d'index localisant leurs émergences, il a le statut d'un interdiscours déclaré, comme on le fait en douane pour les marchandises importées. En forme de catalogue, d'inventaire, de la bibliothèque à laquelle s'adosse le texte, ces « annexes » du texte affichent l'image des relations interdiscursives que celui-ci se reconnaît, dessinant le « paysage »

147 Cf. chap. 5, ex. (25).

148 Cf. chap. 5, ex. (56-58).

149 « Marginales » ou infrapaginales.

150 Cf. ci-dessus 2.4.a les remarques sur Berthier (1998), p. 607.

151 « Périgraphie » dit aussi Compagnon (1979 : 328) de cette « zone intermédiaire entre le horstexte et le texte », à laquelle il consacre des pages suggestives (ibid. : 328-341). 
- dit joliment Compagnon ${ }^{152}$ - « des excursions de l'auteur » dans les ailleurs du déjà-dit ${ }^{153}$.

\subsubsection{Mouvement de la RDA au fil du texte}

L'image d'un texte " par ses autres représentés », telle que la configure sa bordure-interface avec l'extériorité, peut aussi se modifier au cours du texte et cela en fonction de toutes les variables - variant de conserve ou séparément.

\section{(i) Mouvement dans le degré de marquage de la RDA}

Ce mouvement s'observe aisément pour un élément - mot, syntagme - dont les occurrences se succèdent avec un degré de marquage décroissant, signant un progrès dans l'assimilation par le lecteur du terme désormais partagé :

- classique dans les genres relevant peu ou prou de la didacticité, ce mouvement - précisément analysé par S. Moirand (1986), se réalise, dans un texte, par un marquage initial de la ou des premières occurrences d'un élément $\mathrm{X}$ - ils disent « $X$ », ce qu'on appelle « $X$ », « $X$ » comme ils disent, « $X$ »... - qui s'efface aussitôt après, ou peu à peu, touchant, par exemple, des éléments de lexique spécialisé en vulgarisation scientifique, de vocabulaire régional ou étranger dans les guides touristiques, de termes définis à l'orée d'un texte de réflexion comme devant être pris « au sens » d'un certain auteur, de néologismes « branchés » dans la presse féminine...

- il accompagne aussi la construction par le discours d'univers, fictionnels ou de mémoire, dans les romans, souvenirs... sous la forme, commune, du passage de paroles représentées au DD - l a dit : «...X... »- au réemploi ultérieur par $\mathbf{L}$ de l'élément $X$ dont la modalisation doit être reconnue comme modalisation d'emprunt à cette parole antérieure.

Cette figure intratextuelle de l'écho, classiquement entre un DD initial et sa reprise en MAE non explicitée (allusive ou marquée typographiquement), établit de la connivence avec le lecteur, soit dans le partage du jeu rhétorique d'un retour du même en ouverture/clôture d'un bref texte de presse, par là « bouclant » sur

152 Aimant, dit-il, avant de s'engager dans un texte inconnu, en parcourir « l'atlas » pour mesure s’il va s'y trouver « en territoire connu » ou au contraire dépaysé par un déjà-dit qui lui est étranger (p. 333).

153 Sans qu’y soient nécessairement consignés ( $c f$. Berthier ci-dessus p. 607) tous les pas de côté esquissés au fil du trajet central. 
lui-même, soit, en écho lointain, dans la complicité d'une mémoire (textuelle) partagée, cultivée, par exemple, par Proust ${ }^{154}$.

\section{(ii) Mouvement d'ensemble de la bordure dans un texte}

Nombre de réalisations du genre académique de la thèse en sciences humaines manifestent un tel mouvement affectant plusieurs des variables identifiées. Le cheminement classique, menant d'un « état de l'art » (A) sur une question vers son traitement personnel (B), peut se traduire :

- au plan de l'Étendue $\left(\mathrm{V}_{1}\right)$ dont l'importance en (A) diminue sensiblement en (B),

- à celui de la Dispersion $\left(\mathrm{V}_{2}\right)$ des autres convoqués qui, à partir du degré élevé qui caractérise le parcours initial, se focalise sur le ou les ailleurs moins divers que retient l'auteur dans sa démarche,

- à celui de la Tonalité $\left(\mathrm{V}_{5}\right)$ des rapports passant de la plus grande diversité en (A) à un rapport dominant d'appui aux discours choisis en (B),

- à celui de la logique d'émergence du discours autre $\left(\mathrm{V}_{4}\right)$ passant de celle d'attaché à l'objet dont on parle - le champ dont on rend compte - en (A), à celle d'associé au Discours en train de se tenir, en (B), convoquant les autres dont, sélectivement, il s'accompagne, cette variation pouvant se doubler de la double variation $\left(\mathrm{V}_{6}\right)$ d'un DD moins important et de MAE plus nombreuses, dans le passage de la présentation des divers points de vue existants, en (A), à l'appel, en (B), au fil du discours, à des notions, formulations émanant des ailleurs qui le soutiennent.

\subsection{Génétique et histoire du travail des bords.}

Ce mouvement de la RDA est intéressant à observer au plan génétique de la naissance d'un texte, à celui de ses éventuels états ultérieurs, comme à celui de l'évolution d'un auteur ou de la constitution d'un genre.

L'écriture de Flaubert et celle de Montaigne offrent, on le sait, des exemples privilégiés de cette dynamique de la RDA. Chez Flaubert, c'est autant au plan génétique des traces du travail sur les manuscrits qu'à celui de l'évolution depuis les œuvres de jeunesse jusqu'au rêve du Livre « entièrement recopié » que se mani-

$154 C f$. ci-dessus, chap. 11 ex. (20), (21), p. 439 ; pour des exemples de jeux d'échos - proches ou à distance - et notamment les effets qu'en tire Pontalis dans l'évocation de souvenirs donnés à partager dans L'Amour des commencements, cf. Authier-Revuz (1995/2012 : 284-287). 
feste le même mouvement, relevant de la variable $\left(\mathrm{V}_{6}\right)$, allant vers un moindre degré de marquage, c'est-à-dire quant à la séparation entre l'un et les autres, vers l'incertitude et la porosité ${ }^{155}$.

Pour « Montaigne en mouvement » ${ }^{156}$ qui, on l'a dit, pense « avec les autres », c'est une dynamique d'addition qui s'observe d'une strate à l'autre des Essais, à travers leurs éditions successives et les ajouts qu'elles reçoivent, " en marge » (dès lors qu'au rebours de nombre d'éditions contemporaines qui en gomment la dynamique, une édition " multistrate » le donne à lire ${ }^{157}$ ), procédant notamment à l'accueil "d'autres autres » enrichissant toujours $\left(\mathrm{V}_{1}\right)$ la bordure du texte.

Évoquons aussi le mouvement que dégage Godard (2011) ${ }^{158}$ dans l'écriture de Céline, entre le Voyage au bout de la nuit et Mort à crédit : celui - relevant des variables de l'Étendue $\left(\mathrm{V}_{1}\right)$ ou du degré de marquage des formes $\left(\mathrm{V}_{6}\right)$ - d'une raréfaction du signalement typographique des MAE de mise à distance des «mots d'un certain discours social » (« moment d'égarement », « honneur de la famille »...), et dans lequel le critique voit « un aspect peu visible mais important de la mutation du style de Céline » vers un « plurivocalisme » de «partage intime de l'individu » ignorant la frontière des guillemets.

Ou encore l'évolution que Herman (2008) met au jour au fil des discours de De Gaulle de 1940 à 1945, touchant le type et l'identité $\left(V_{3}\right)$ des autres convoqués, témoignant d'un travail rhétorique de « présentation de soi », « modulé » dans la durée ${ }^{159}$.

Et enfin, dans le cadre d'une réflexion collective sur l'espace générique des Discours " tenant lieu » d'un autre discours ${ }^{160}$, les observations de D. Mazzuchetti ou de F. Sitri ${ }^{161}$ faisant apparaître la double dimension temporelle qui les traverse : celle, pour le premier, de l'histoire de la genèse normalisante et de la variation des prescriptions qui les régissent (notamment quant à la Forme (V6) de

$155 C f$. par exemple (dans l'immense corpus des études flaubertiennes) la minutieuse étude génétique de la RDA que comporte le travail de Funakoshi-Teramoto (2005), notamment p. 233-257, ou dans Herschberg-Pierrot (1981) le tracé de l'évolution de la « stéréotypie » dans l'ensemble de l'œuvre.

156 Pour reprendre le titre de l'ouvrage de Starobinski.

157 Pour un survol efficace, $c f$. Compagnon (2013 : 153-173).

158 Au chapitre « Le plurivocalisme célinien », p. 138-140 notamment.

159 Ainsi que le souligne Amossy (2010 : 154-155) évoquant à ce propos « l'axe temporel » sur lequel, « en tenant compte de toutes les voix qui interviennent dans cette opération complexe », se construit « une image de soi ».

160 Cf. ci-dessus 1.1.1, p. 583.

161 Mazzuchetti (à paraître), Sitri (à paraître). 
RDA à mettre en œuvre) ; celle, pour la seconde, dans la sphère universitaire, du travail génétique inattendu et éclairant - brouillons, corrections... - sous-jacent à un compte rendu de conseil d'université.

\section{Pour finir...}

Approcher les Discours «par leurs bords », c'est voir apparaître, témoignant de leur manière propre « d'être avec », une fascinante diversité de bordures :

- $\quad$ larges bandes, couches épaisses... minces lisières, étroites marges...

- riches, encombrantes, envahissantes... pauvres, discrètes, légères...

- $\quad$ solides, consistantes... incertaines, poreuses...

- $\quad$ à bords tranchés, nets, raides... flous, fondus, fragiles...

- qui renforcent, soutiennent, étayent... qui aèrent, ouvrent, respirent...

- qui protègent, rassurent, abritent... qui bousculent, dispersent, disséminent...

- prescrites, attendues, attentives..., imprévues, fantaisistes, désinvoltes...

- $\quad$ sereines, calmes, neutres... agitées, turbulentes, passionnées...

- bordures comme des rings ou des plateaux d'escrime, polémiques, ironiques, persiflantes, agressives, hargneuses, haineuses, assassines...

- bordures comme des terrains de jeu, pour des jeux de pistes, de masques, de devinettes, des puzzles, des carnavals de citations détournées ou loufoquement attribuées...

- $\quad$ bordures comme des défilés de pancartes, de slogans, d'effigies, d'icones...

- bordures comme des lieux dédiés à des échanges en bonne compagnie, aimables, complices, respectueux, nourriciers...

Me retournant - dans un redoublement métadiscursif - sur la bordure dont j'ai « cerclé » mon propre discours dans cet ouvrage, c'est le soupçon d'un excès - reconnaissance de dette, cheminement par confrontation, recherche d'appuis... - qui apparaît. J’y répondrai par des citations encore, empruntées :

- à Michel Schneider dans Morts imaginaires,

On jugera peut-être ces pages trop érudites et grevées de dettes citationnelles. Je ne m'en excuse pas. On est ce qu'on doit aux autres. Et je tiens à mes lectures : ce sont elles qui me font écrire (Schneider, $2003:$ 20),

- à Françoise Armengaud dans Devoir citer [...],

Citer l'autre est bien l'une des multiples manières de vivre avec lui (Armengaud (2005 : 24), 
- à Jean Starobinski enfin dans La Parole est moitié à celui qui parle,

Je ne suis pas solipsiste et je pense en société. Je crois même qu'une vraie recherche ne commence que lorsqu'on se sent en compagnie (p. 45) ${ }^{162}$.

162 La Parole est moitié à celui qui parle - Entretiens avec Gérard Macé, 2009, La Dogana, Chênebourg. 